\title{
MEGARA spectrograph mechanics and opto-mechanics in the AIV phase
}

\author{
Maldonado-Medina, Manuel *a , Pérez-Calpena, Ana ${ }^{a}$, García-Vargas, María Luisa ${ }^{\mathrm{a}}$, Sánchez- \\ Blanco, Ernesto $^{\mathrm{a}}$, Martínez Delgado, Ismael ${ }^{\mathrm{a}}$ Gil de Paz, Armando ${ }^{\mathrm{b}}$, Arrillaga Echaniz, Xabier ${ }^{\mathrm{c}}$ \\ Gallego Maestro, Jesús ${ }^{\mathrm{b}}$, Carrasco Licea, Esperanza ${ }^{\mathrm{d}}$, Iglesias-Páramo, Jorge ${ }^{\mathrm{e}}$, Sánchez Moreno, \\ Francisco $^{\mathrm{f}}(+)$ \& MEGARA Team \\ ${ }^{a}$ FRACTAL SLNE (Madrid, Spain), ${ }^{b}$ Universidad Complutense de Madrid (Madrid, Spain), ${ }^{\mathrm{c}}$ AVS \\ (Elgoibar, Spain), ${ }^{\mathrm{d}}$ NAOE (Puebla, México), ${ }^{\mathrm{e}}$ Instituto de Astrofísica de Andalucía IAA-CSIC \\ (Granada, Spain), ${ }^{\mathrm{f}}$ Universidad Politécnica de Madrid (Madrid, Spain),
}

\begin{abstract}
MEGARA (Multi-Espectrógrafo en GTC de Alta Resolución para Astronomía) is the future optical Integral-Field Unit (IFU) and Multi-Object Spectrograph (MOS) for the GTC $10.4 \mathrm{~m}$ telescope. The spectrograph is currently being integrated in the laboratory for a pre-shipping review in September 2016. This paper presents the current status and final performance of the spectrograph mechanics and opto-mechanics, composed of the mechanisms and the large optomechanical elements mounts.
\end{abstract}

Keywords: MEGARA, GTC, mechanical design, mechanism, spectrograph, opto-mechanics, mechanism

\section{INTRODUCTION}

MEGARA (Multi-Espectrógrafo en GTC de Alta Resolución para Astronomía) is a fiber-fed spectrograph with an optical Integral-Field Unit (IFU) and a set of robotic positioners for Multi-Object Spectroscopy (MOS) that will be installed at Folded-Cassegrain focus of the GTC (Gran Telescopio CANARIAS) 10.4-m telescope. The fibers will feed a spectrograph to be placed on the GTC Nasmyth platform.

The MEGARA IFU mode will offer a fiber bundle covering 12.5 " x 11.3 " with a spaxel size of 0.62 ". The MEGARA MOS will allow observing up to 100 objects thanks to the MEGARA MOS positioners that can place a mini-bundle of 7 fibers $\left(0.62\right.$ '/fiber) covering an area of 1.6 " on the sky in a region of $3.5^{\prime}$ x $3.5^{\prime}$ around the IFU.

The IFUs and the 100 positioners that constitute the Fiber MOS shall be installed at a GTC Folded Cassegrain focal station. Then, IFU and MOS fibers bundles shall be routed through the telescope structure and rotators to the Spectrograph entrance.

A mechanism placed at the pseudo-slit position allows exchanging the observing modes and also acts as focusing mechanism. The spectrograph is a collimator-camera system that has a total of 11 VPHs simultaneously that are placed in the pupil by means of a large rotating wheel and an insertion mechanism. The LN2 bath cryostat hosts an E2V 231-84 $4 \mathrm{kx} 4 \mathrm{k}$ CCD.

In the following sections we describe the current status of the different mechanical and opto-mechanical elements taking as a guide the integration and alignment procedure.

*manuel.maldonado@fractal-es.com

Ground-based and Airborne Instrumentation for Astronomy VI, edited by Christopher J. Evans, Luc Simard, Hideki Takami Proc. of SPIE Vol. 9908, 99088S · C 2016 SPIE · CCC code: 0277-786X/16/\$18 · doi: 10.1117/12.2233041 


\section{OVERALL DESCRIPTION OF THE SPECTROGRAPH}

In the next pictures we show the 3D view of the detailed design of the MEGARA spectrograph. The positions of the pseudo-slits, the collimator with the shutter, the VPH in use (located at the instrument pupil), the camera and the cryostat are labeled below. A major fraction of the area of the MEGARA spectrograph $3 \mathrm{~m} \times 2 \mathrm{~m}$ optical table is occupied by the VPH wheel, where are total of 11 VPHs can be placed.

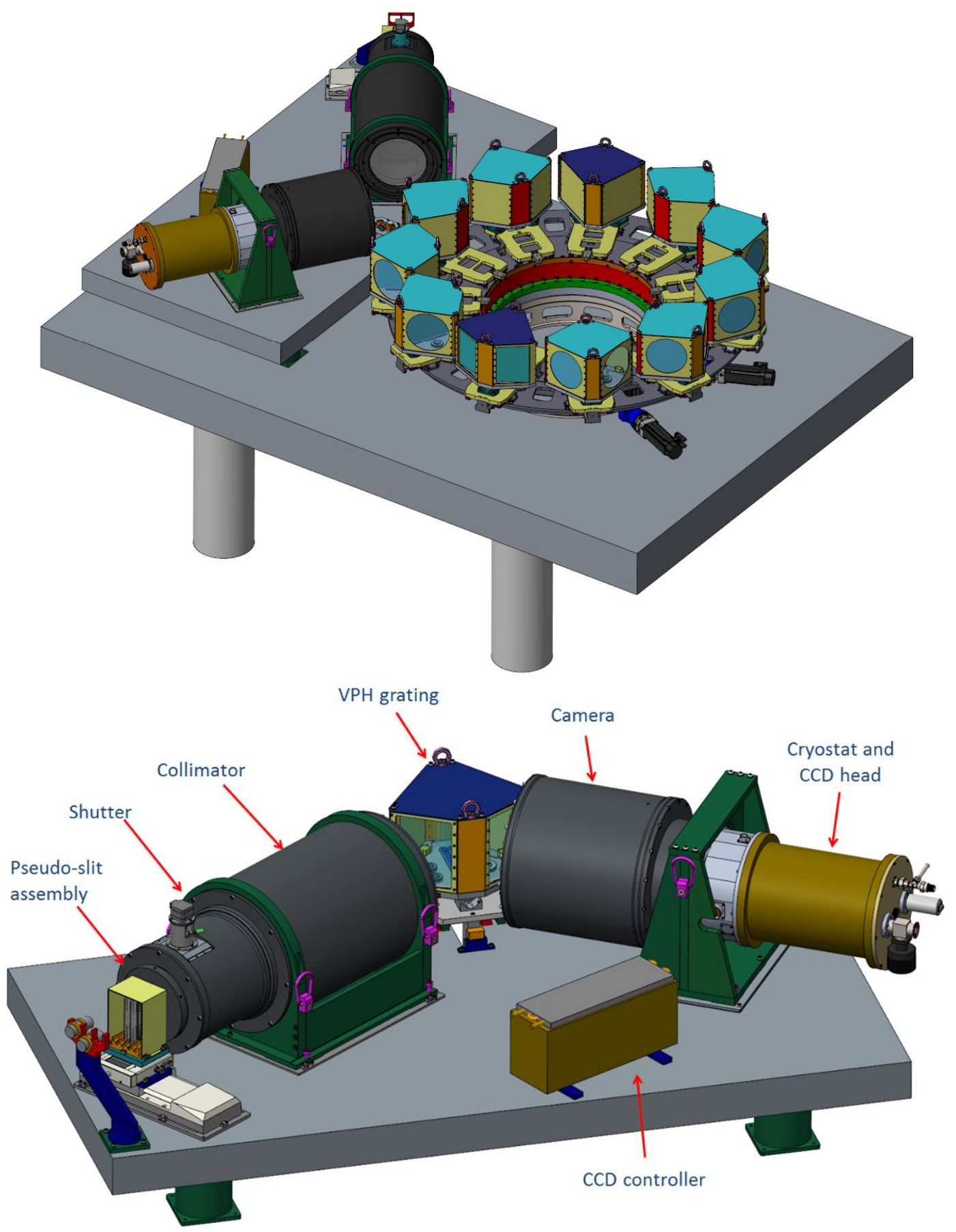

Figure 1: MEGARA spectrograph overall view with labels on the main optical path elements 


\section{SPECTOGRAPH MECHANICS SUBSYSTEMS UPDATES}

\subsection{Optical Bench}

The whole spectrograph is located on a Newport optical table (Custom M-RPR 2000 x 3000 x $203 \mathrm{~mm}$ ). These tables provide high structural stiffness and broadband damping.

On this table there is a smaller $1 \mathrm{~m} \times 2 \mathrm{~m}$ optical table (called auxiliary table) where all functional elements but the VPHs wheel are located. This table was manufactured by TMC (model TMC 78-244-12R). Four custom manufactured stainless steel supports locate this table $268 \mathrm{~mm}$ above the surface of the main table. This avoids having large optical mounts for the lenses and allows the location of the VPH insertion mechanism that will be described later on.

The table was originally supported by 4 isolating legs (Newport LabLegs SL-1200) but during the las phase of the design we decided to add another leg at the center of the table, due to the extra weight introduced by the final design and manufacturing of the wheel's assembly and the new design of the spectrograph cover.

\subsubsection{Status}

The main table was installed early at the UCM LICA laboratory. The auxiliary table was installed past September, since a custom cut had to be done in the table to allow the insertion mechanism to reach the pupil.

In Figure 2, we see both tables at the LICA tab.

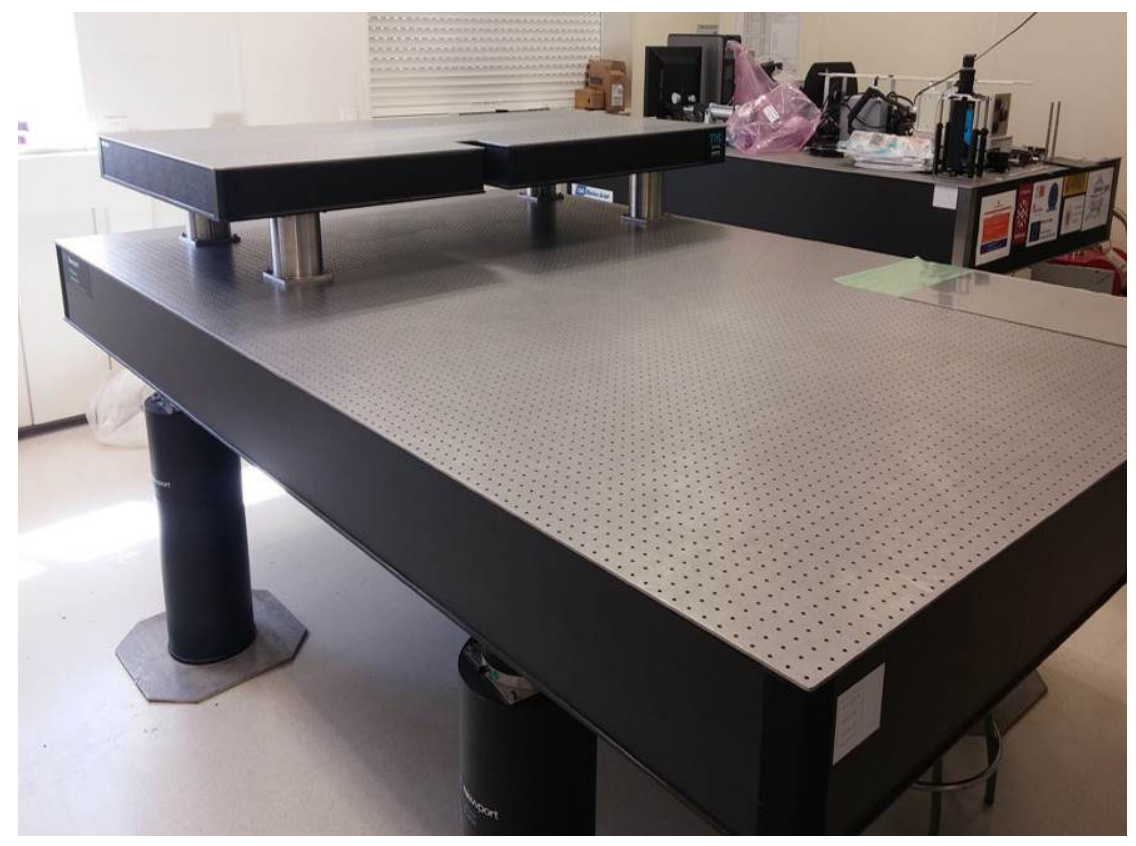

Figure 2: Both main and auxiliary optical tables at the UCM LICA lab. The steel plates on the floor protect the tiles and spread the load.

\subsection{System integration procedure}

In the spectrograph there are four main opto-mechanical units: slit, collimator, gratings wheel and camera. The heaviest and bulkiest unit is the grating wheel. This unit was first installed and fixed on the optical bench. Fixed targets placed into this unit were the reference system for all the assembly procedure and all the alignment verification. The alignment axis defined by these targets was transferred to an alignment telescope that became the reference tool for the procedure. 


\subsection{VPH gratings wheel}

The first element to be installed in the optical table was the wheel.

The spectrograph includes the capability of the automatic interchange of $11 \mathrm{VPH}$ gratings that are placed on several platforms located in a large wheel. Each platform has a pair of guideways that are inserted on their corresponding carriages that are screwed to the wheel. The VPH gratings mounts are easily installed and removed from the wheel, and any VPH can be installed on any platform, on any wheel position.

After rotating the wheel with precision servomotors, the VPH of interest in inserted on the pupil position of the optical path with an insertion actuator.
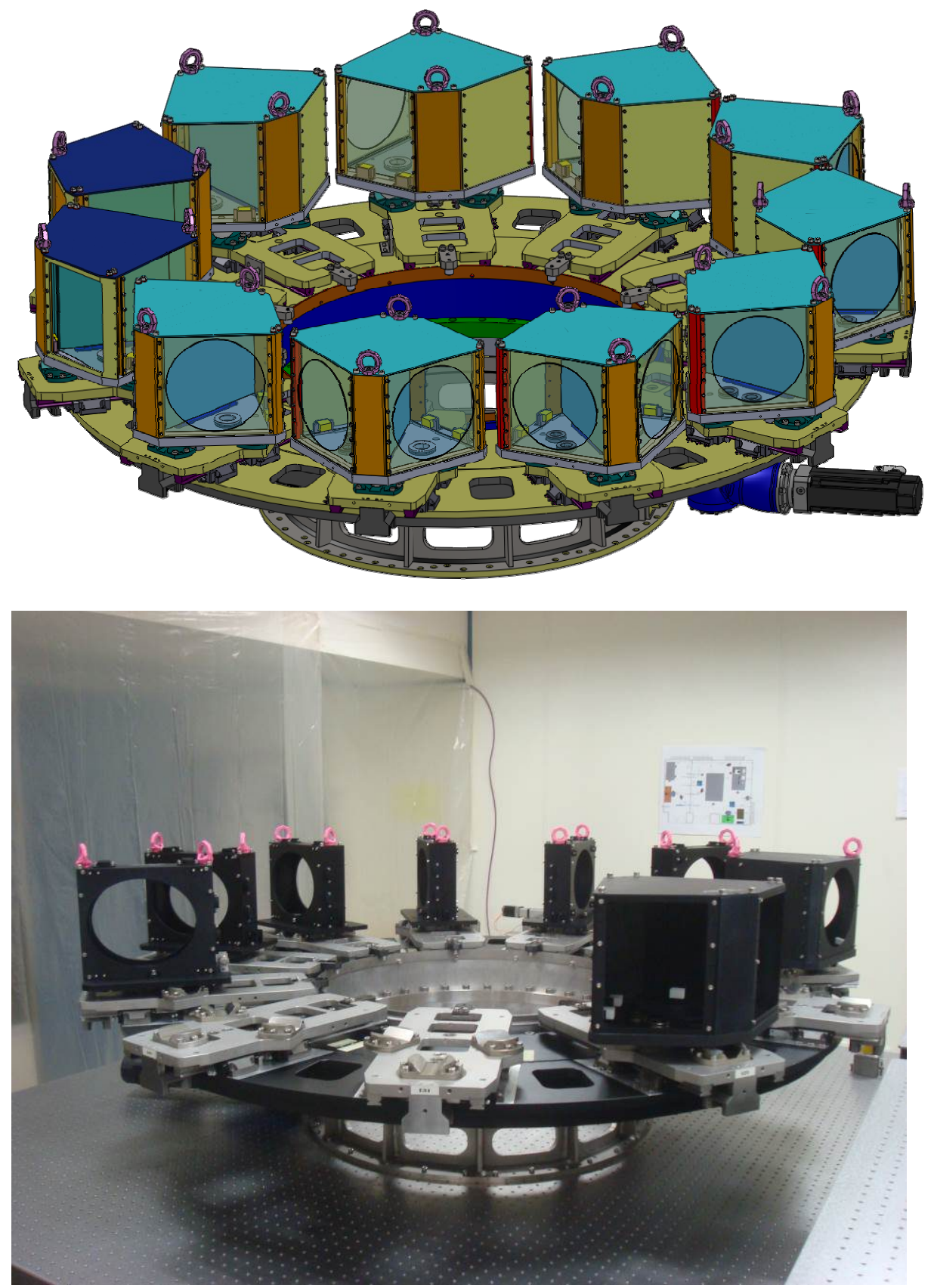

Figure 3: CAD model of the VPH wheel assembly and photo (taken past November 2015 during testing) 
In the wheel assembly we can distinguish 4 elements: the base, the bearing, the wheel itself and the platforms. All of these are properly screwed to its neighbor.

\section{The wheel}

The wheel is a welded structure made of aluminum alloy 5083. Its weight is $89 \mathrm{~kg}$. Its outer diameter is $\varnothing 1517 \mathrm{~mm}$ and the central hole is $\varnothing 739 \mathrm{~mm}$. The top plate is $15 \mathrm{~mm}$ thick. It has 22 radial stiffeners $10 \mathrm{~mm}$ wide located on the center line of the carriages plus 2 diametral stiffeners: one at the outer edge of the wheel $\varnothing 1470$ and other in the center (both $10 \mathrm{~mm}$ wide). Continuous weld has been defined in the main parts and windows have been added to reduce weight. It has counterbored holes for the attachment to the bearing (with M12X100 DIN 912 screws). It has a radial fitting diameter for the bearing and a pattern of M5 threaded holes for the attachment of the carriages of the linear guides.

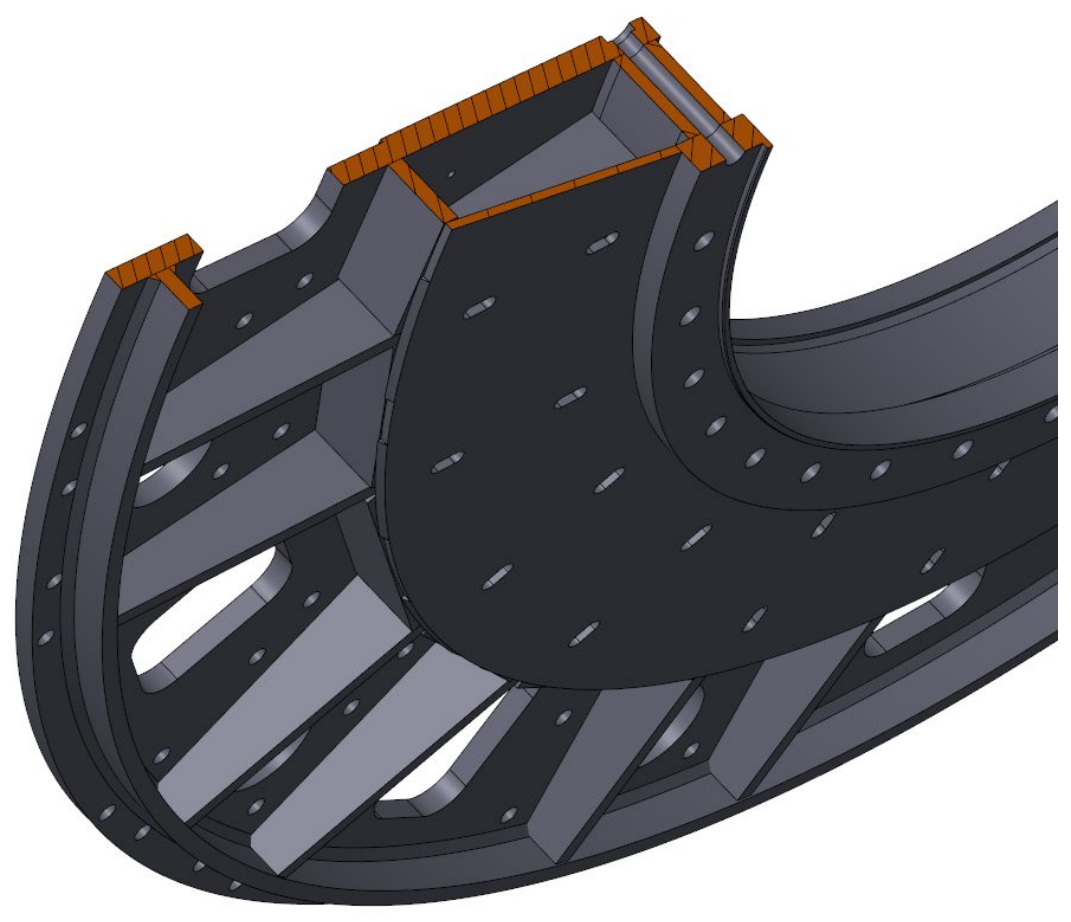

Figure 4: CAD model of the VPH wheel

\section{The support structure}

The support structure is a stainless steel (AISI 316) open cylinder welded structure, with external and internal diameters of $\varnothing 770 \mathrm{~mm}$ and $\varnothing 661 \mathrm{~mm}$ respectively, with a height of $142.5 \mathrm{~mm}$, attached to a circular plate base. Pockets were mechanized to reduce weight. It also includes 2 flat surfaces for the attachment of the motors 


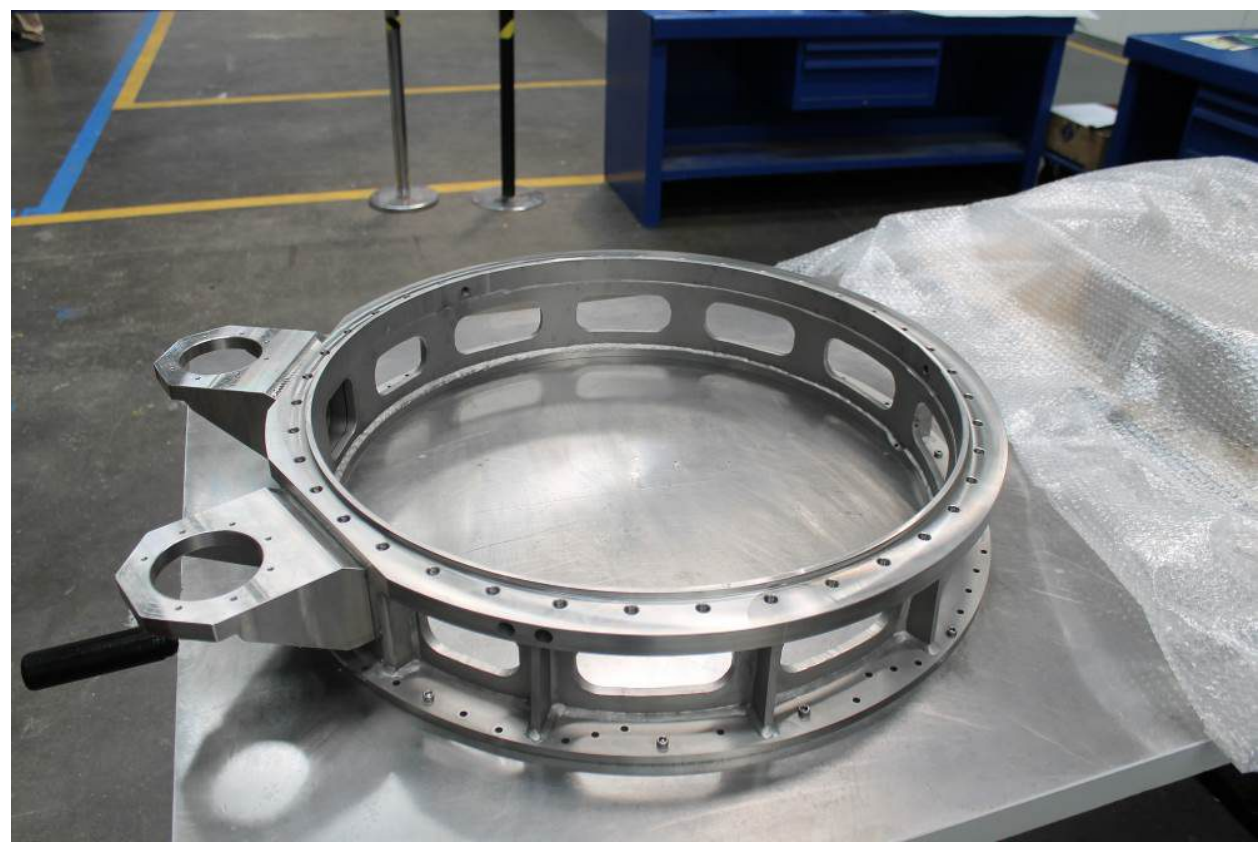

Figure 5: Support structure of the wheel assembly

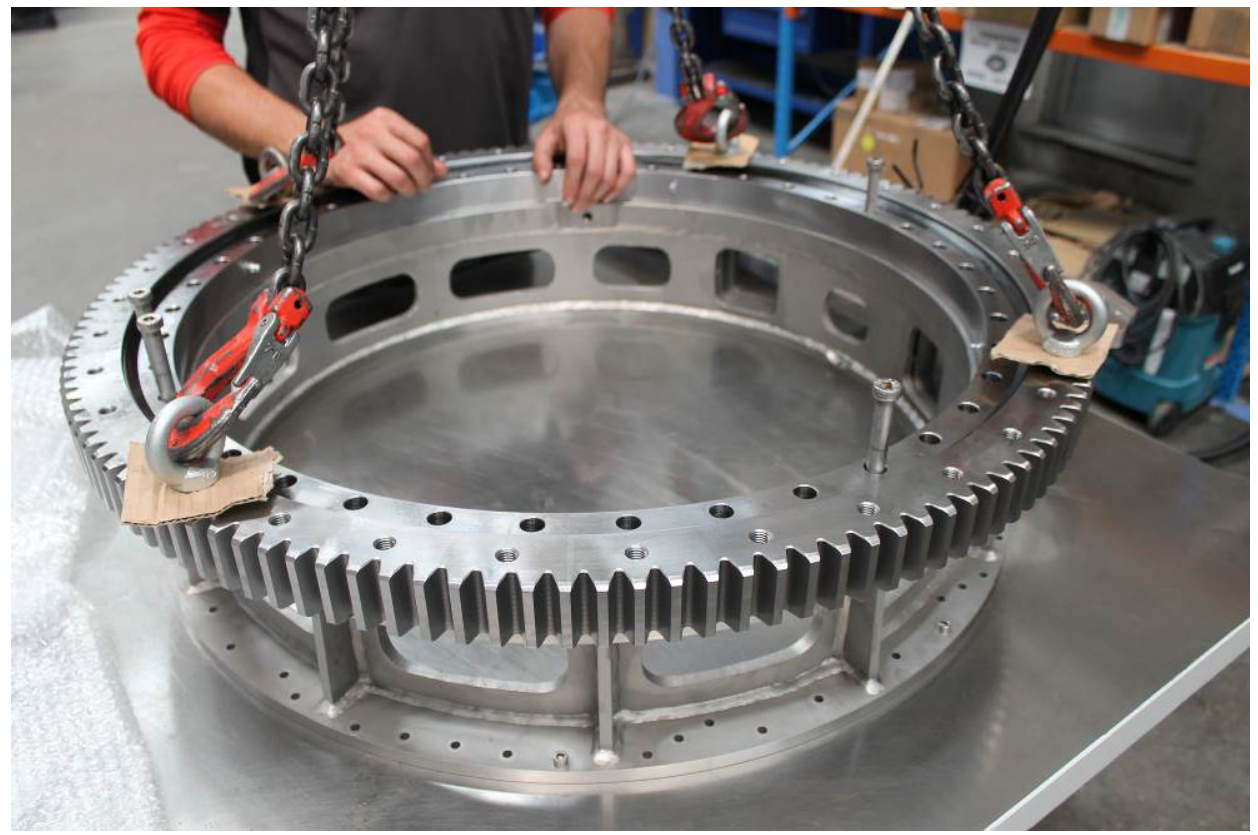

Figure 6: Bearing of the wheel assembly

\section{The bearing}

The size of the wheel determines the diameter of the bearing. The load capacity of such large bearings exceeds our requirements. It have been selected the INA crossed rolled bearing XSA140744 with external gear teeth. The weight of the bearing is $59 \mathrm{Kg}$. 


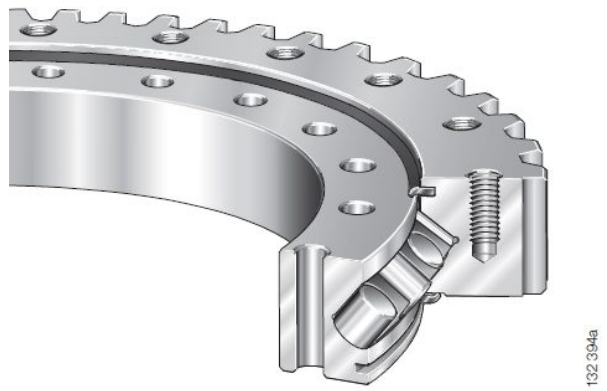

Figure 7: INA crossed roller bearing XSA

\section{The platforms}

The platforms that carry the VPH gratings are made of AISI $316 \mathrm{~L}$. They were given a micro shot penning surface treatment to reduce reflections. Their size is $449 \mathrm{~mm}$ long, $18 \mathrm{~mm}$ thick and $249 \mathrm{~mm}$ wide at its maximum. The outer shape and internal pockets have been optimized to reduce weight.

On the surface of the platforms there is a 3-groove kinematic support configuration to locate the spheres that are used to install the VPH gratings mounts. These are 3 separate parts made of AISI 440C hardened steel (>58 HRC). This configuration maintains the VPH center stable during temperature variations and provides good repeatability. Each VPH mount type should be properly aligned with respect to the 3 spheres, and any VPH can be installed on any platform.

Each platform has a pair of guideways screwed on its downside and these guideways are inserted on their corresponding carriages that are screwed to the wheel.

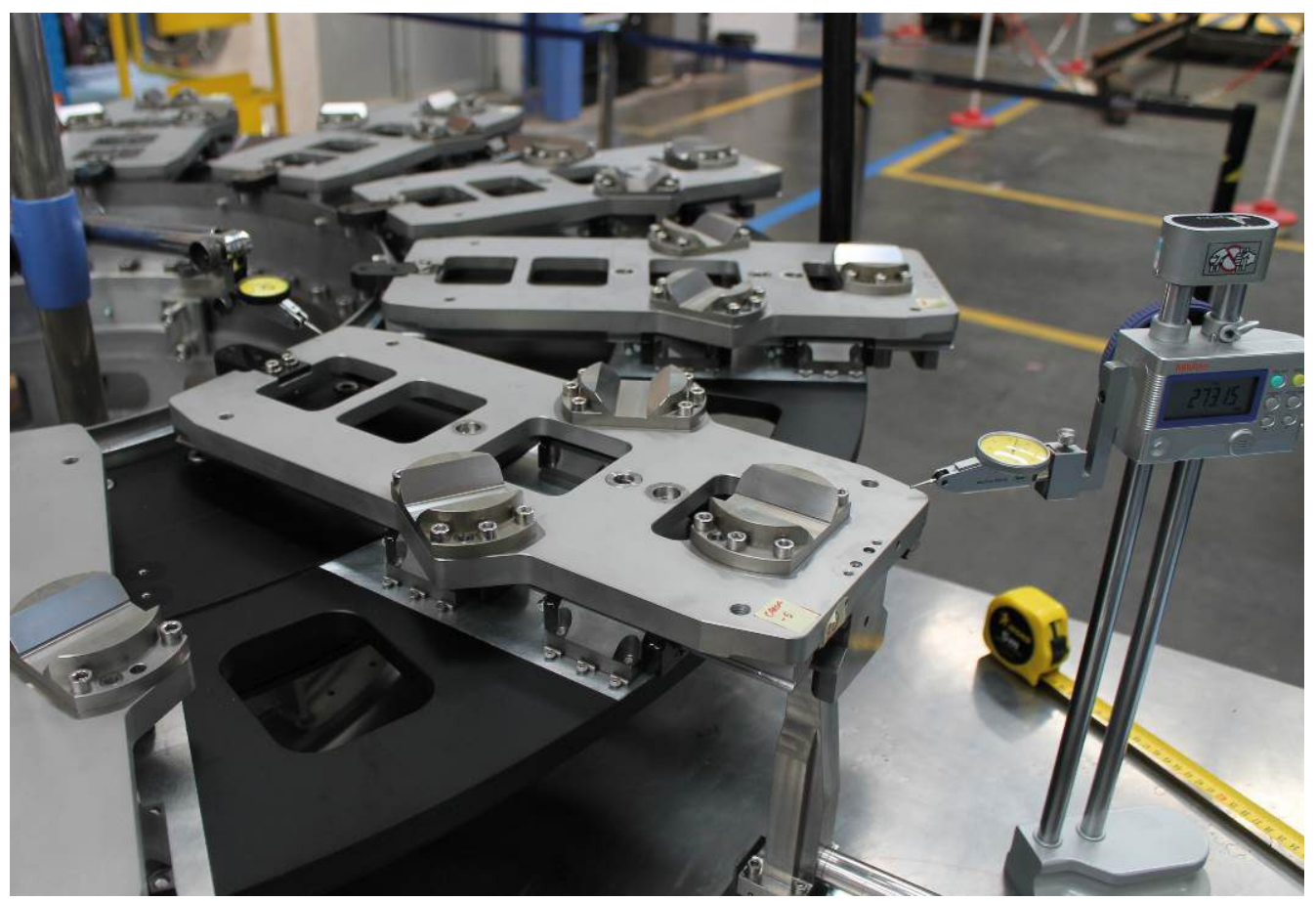

Figure 8: Image of one of the platforms during testing 


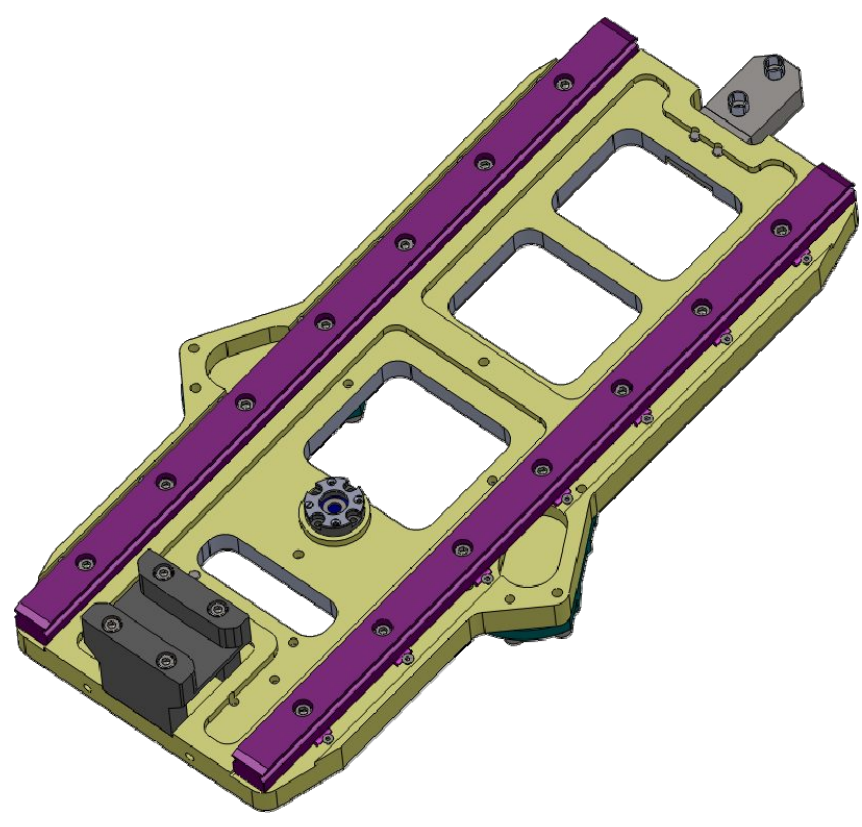

Figure 9: Detail of the CAD model of the underside of the platform

It has been designed a preloading mechanism in order to generate a vertical $50 \mathrm{~N}$ force to fix the VPH gratings mount to the platform. It has been located in the center of the 3 spheres and prevents any accidental displacement of the mount away from its location. The location of the mechanism will leave freedom to the expansion or contraction of the system.

\section{Wheel actuation}

The actuators proposed for driving the wheel are a pair of servomotors with planetary reducers preloaded on a crossed rolled bearing gear by pinions in order to remove backlash. The actuators are made up of a servomotor with brake attached to a planetary reducer and a pinion. The control electronics (digital) shall control the preload in real time. For this reason, both servomotors shall include an encoder on axis; both motors should have an absolute encoder although one shall work as master and the other one as slave.

The servomotors selected are from the Kollmorgen Synchronous servomotors of the AKM series (Brushless three-phase) model AKM 33C $-\mathrm{ABC} 2 \mathrm{DB} 00$ which provides $2.54 \mathrm{~N} \cdot \mathrm{m}$ rated torque at $2000 \mathrm{rpm}$.

The encoder is placed on the motor shaft. The selected encoder is an encoder type EQN1125 from Heidenhain, with 512 line counts per revolutions and an absolute signal of 8192 position values. The motor controller is S70162 from Kollmorgen.

The reducer is the WITTENSTEIN TK+010S-MF2-100-5C1, with a reduction ratio of 100 . These helical planetary gearheads have a nominal output torque of $40 \mathrm{~N} \cdot \mathrm{m}$ at $4500 \mathrm{rpm}$ (nominal output speed).

A testing campaign was done at the LICA lab to verify the precision and repeatability of the mechanism. It met and exceeded the requirements of $0.01^{\circ}$. 


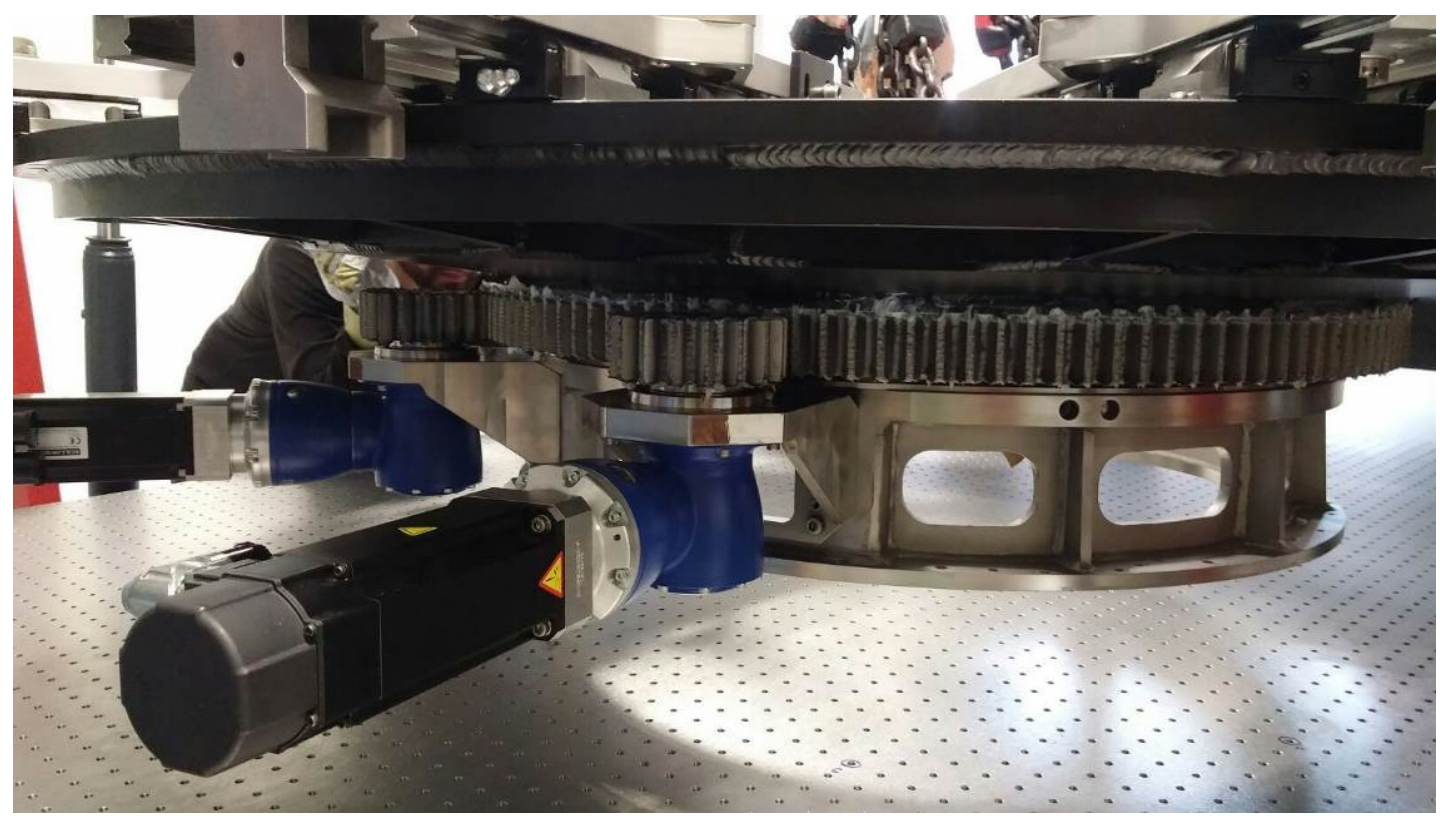

Figure 10: View of the wheel assembly during its integration, with the motors, reducer and bearing clearly visible.

\subsection{Insertion mechanism}

The insertion mechanism consists of an electro-mechanical actuator that translates a stud type track roller that moves on a railway. As the wheel rotates, platform protrusions are passing by the roller during grating selection. Once the desired VPH is located on position, this track roller engages the protrusion of the VPH mount platform and pulls it to the optical path or pushes it out of it. The angular position of the VPH with respect to the optical path is defined with the rotation of the wheel and the lateral position is determined by the elongation of the actuator.

The actuator is model PC32LX-999-B10-0250-FM from Thomson. The stepper motor is model CTM22NLF31 from Danaher motion. The parked position and the observing position are defined using micro-switches.

The mechanism was tested and met and exceeded the requirement of $\pm 0.1 \mathrm{~mm}$. 


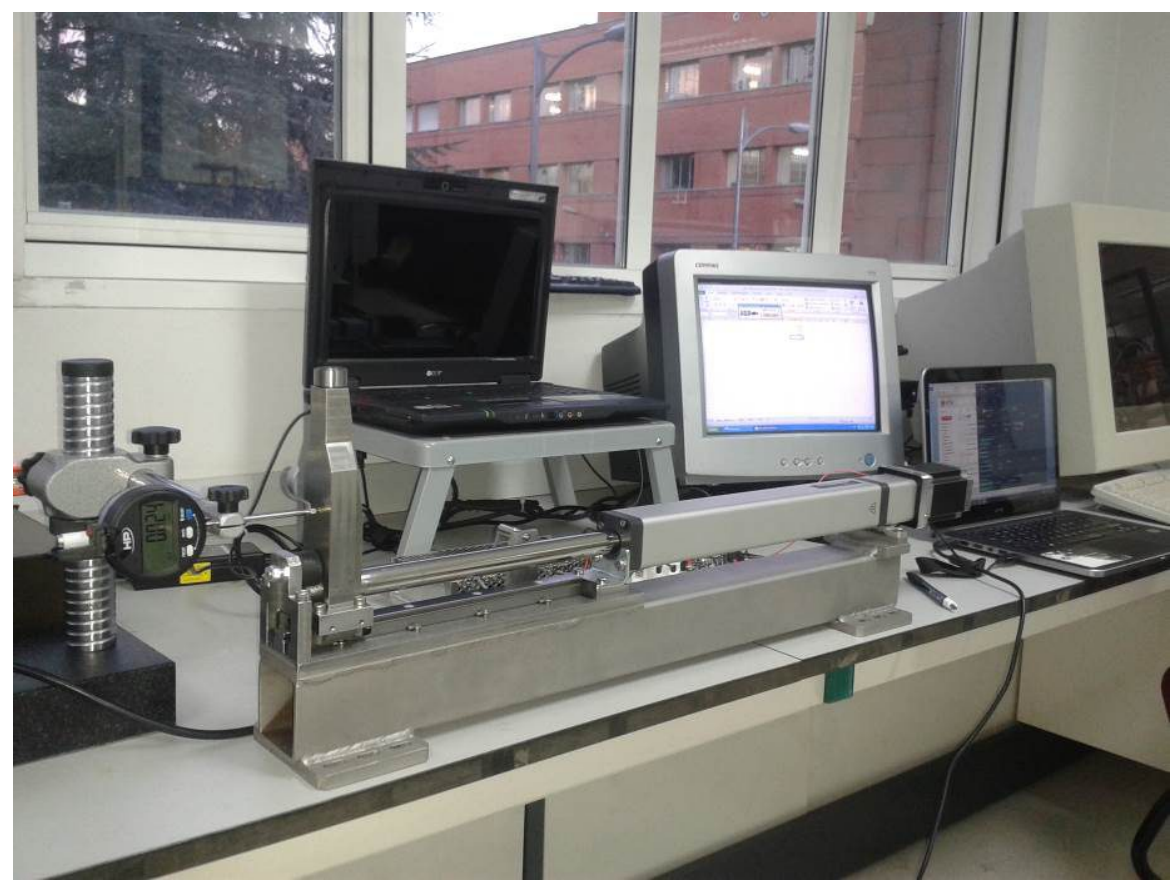

Figure 11: Image of the insertion mechanism testing

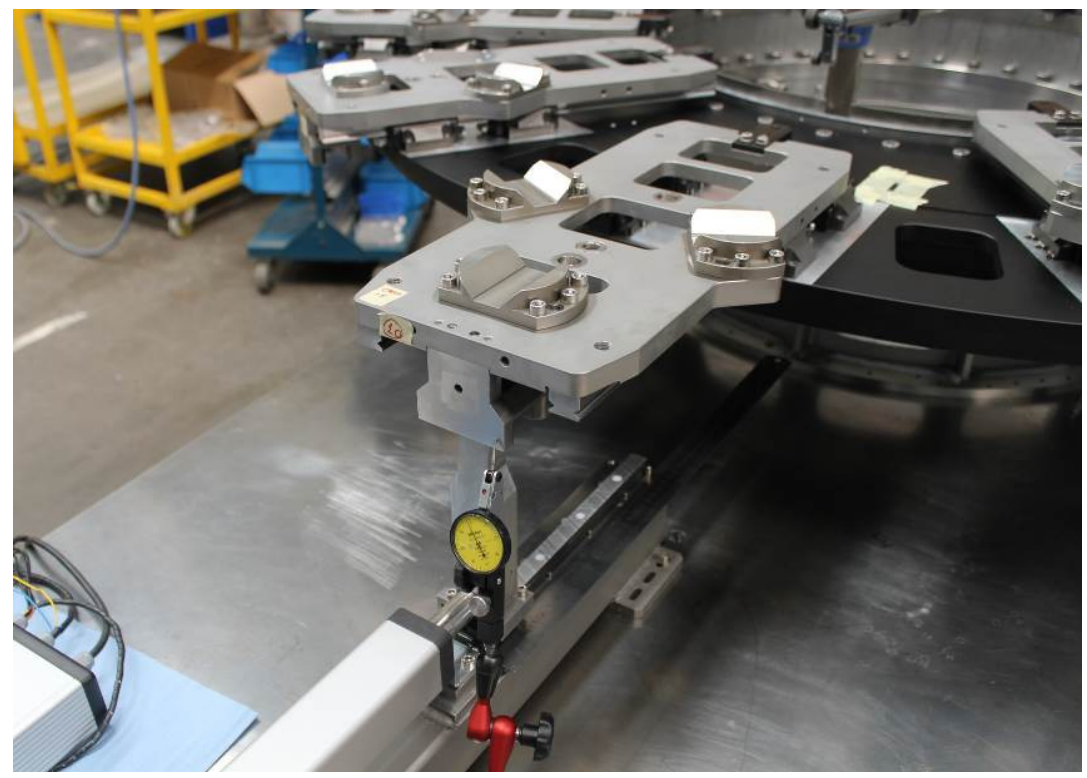

Figure 12: Image of the insertion mechanism during testing carrying a platform

The mechanism was installed on its position under the auxiliary table past month and undergone more successful tests. 


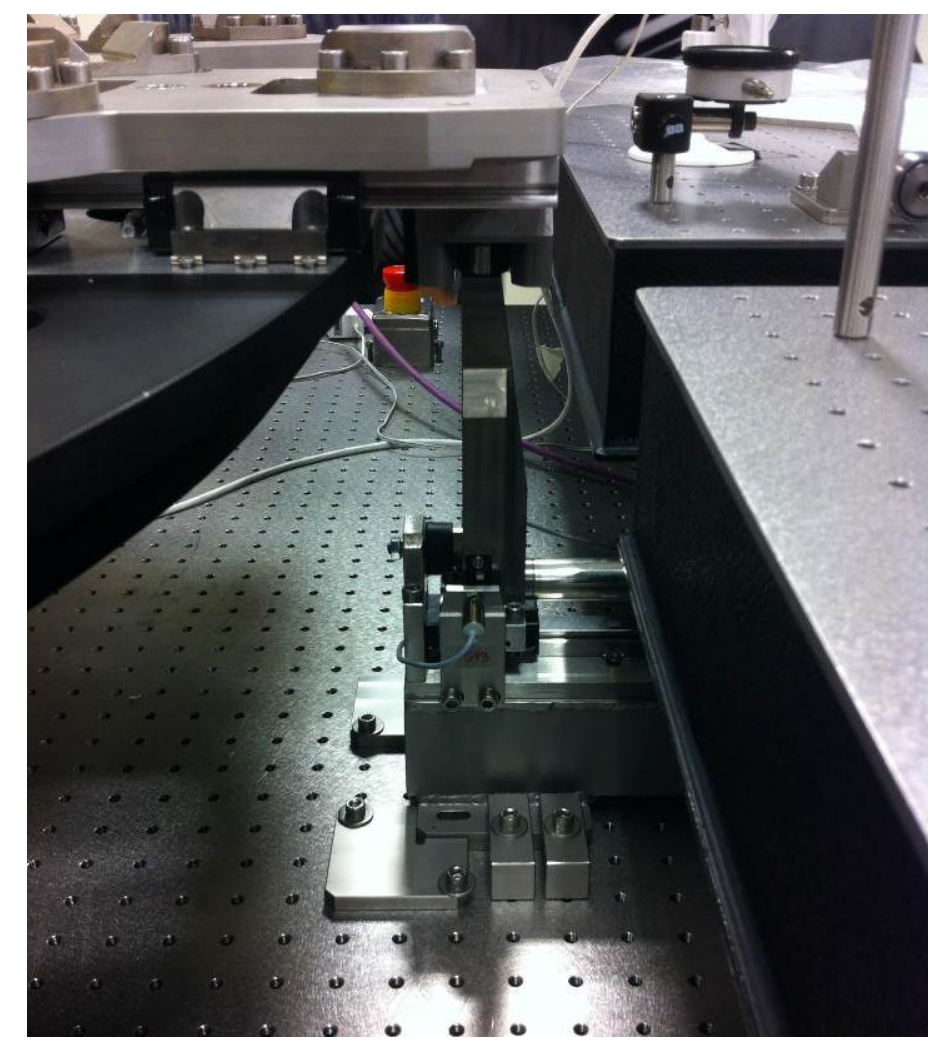

Figure 13: Image of the insertion mechanism on its final location under the auxiliary table

\subsection{VPH gratings mounts}

In MEGARA spectrograph, different types of pupil elements (all of them based on VPH-type gratings) can be accommodated in the pupil position to provide the different spectral modes, with resolution power ranging between 5,500 and 17,000. There will be 6 LR, 10 MR and 2 HR mounts available.

Low-resolution units can be built with simple gratings sandwiched between two flat windows; mid-resolution and highresolution units are provided with the gratings sandwiched between flat windows and two symmetric prisms that allow the beam to incident on the VPH in the optimum angle.

We have seen that the opto-mechanical mounts of the VPHs are located in the platforms of the wheel with a three-ballson-three-grooves kinematic support. Here we describe the mounts and see their status.

\subsubsection{Design of the opto-mechanical mount of the low resolution (LR) VPH}

The VPH grating sandwich must be held with a mount that guarantee its stability during the observation and with temperature changes between different observations. For this reason and to avoid the stress on the gelatine, one of the windows is fixed to the mount and the other is floating and preloaded with springs. On Figure 14 the blue parts are the spring locations. Figure 16 shows the configuration of the spring supports and the location of the hard supports. 


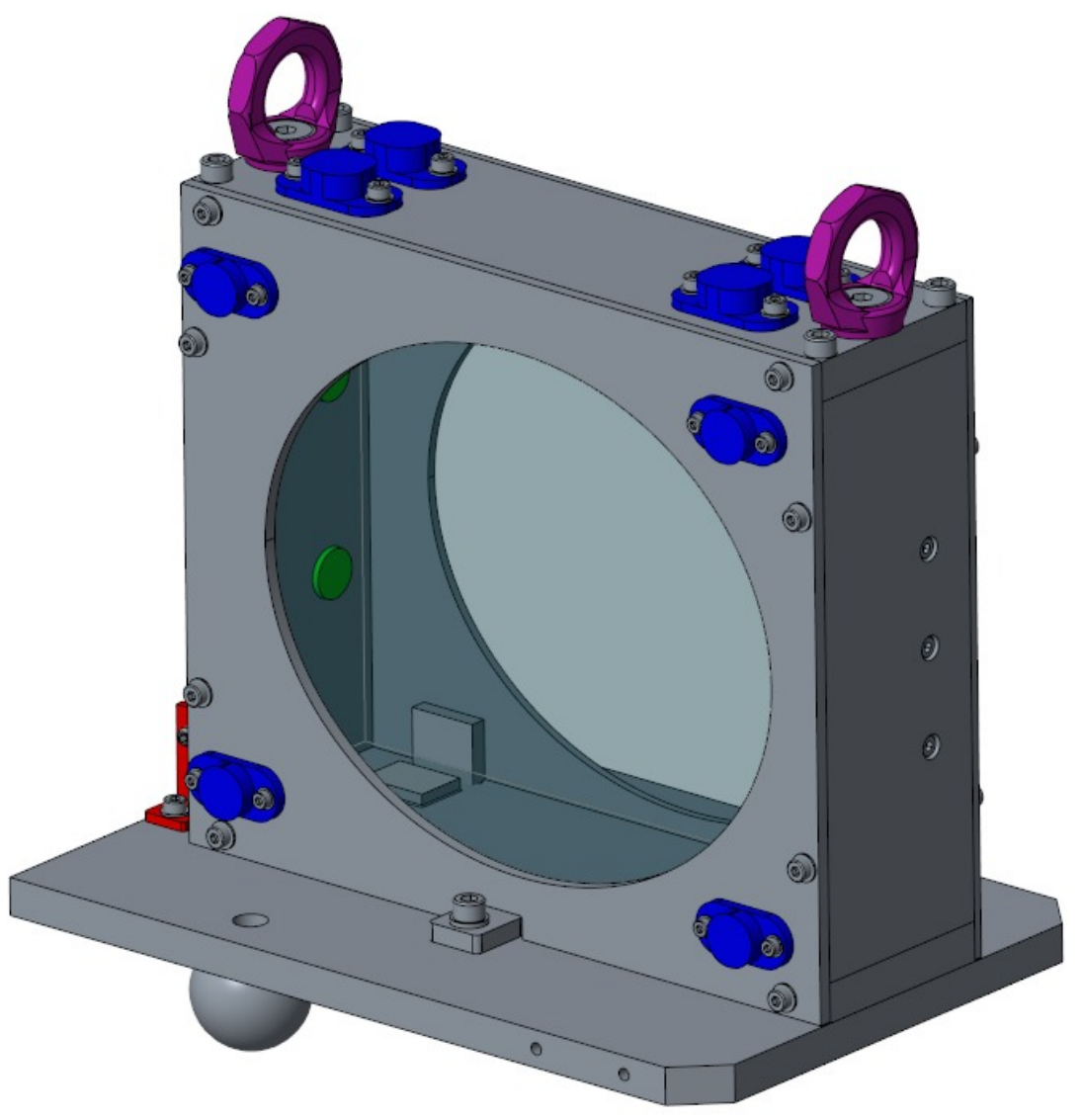

Figure 14: 3D CAD of the low resolution VPH mount

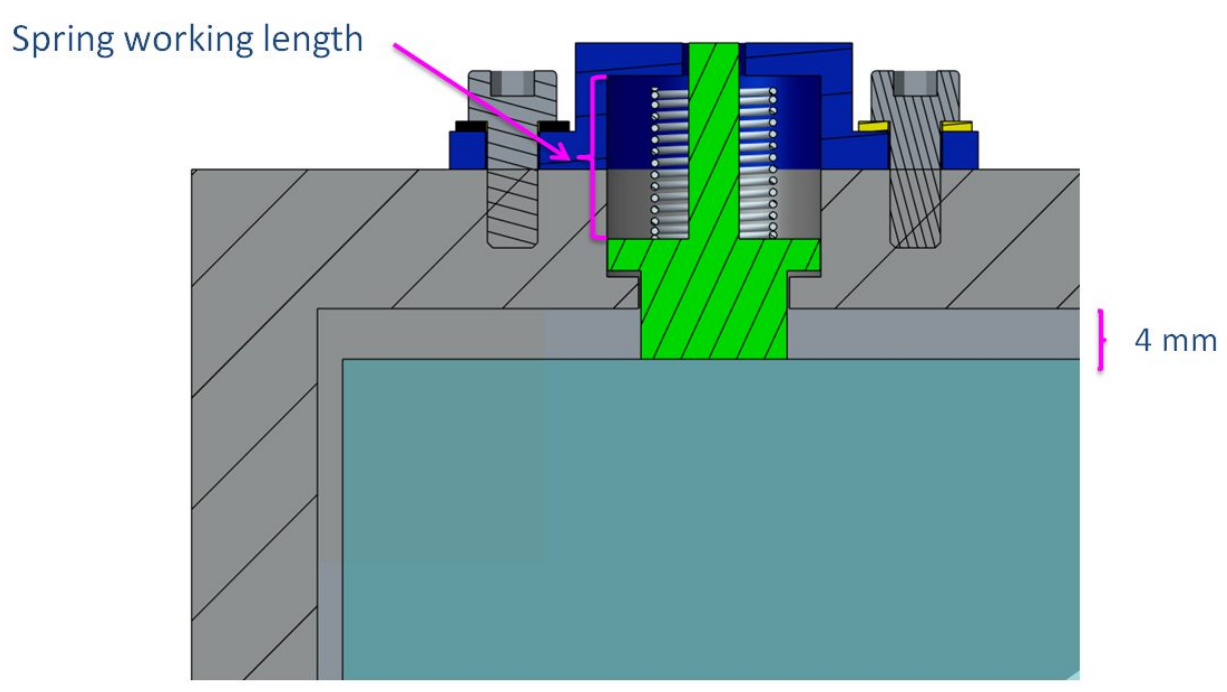

Figure 15: Details of the spring supports of the low resolution VPH mount 


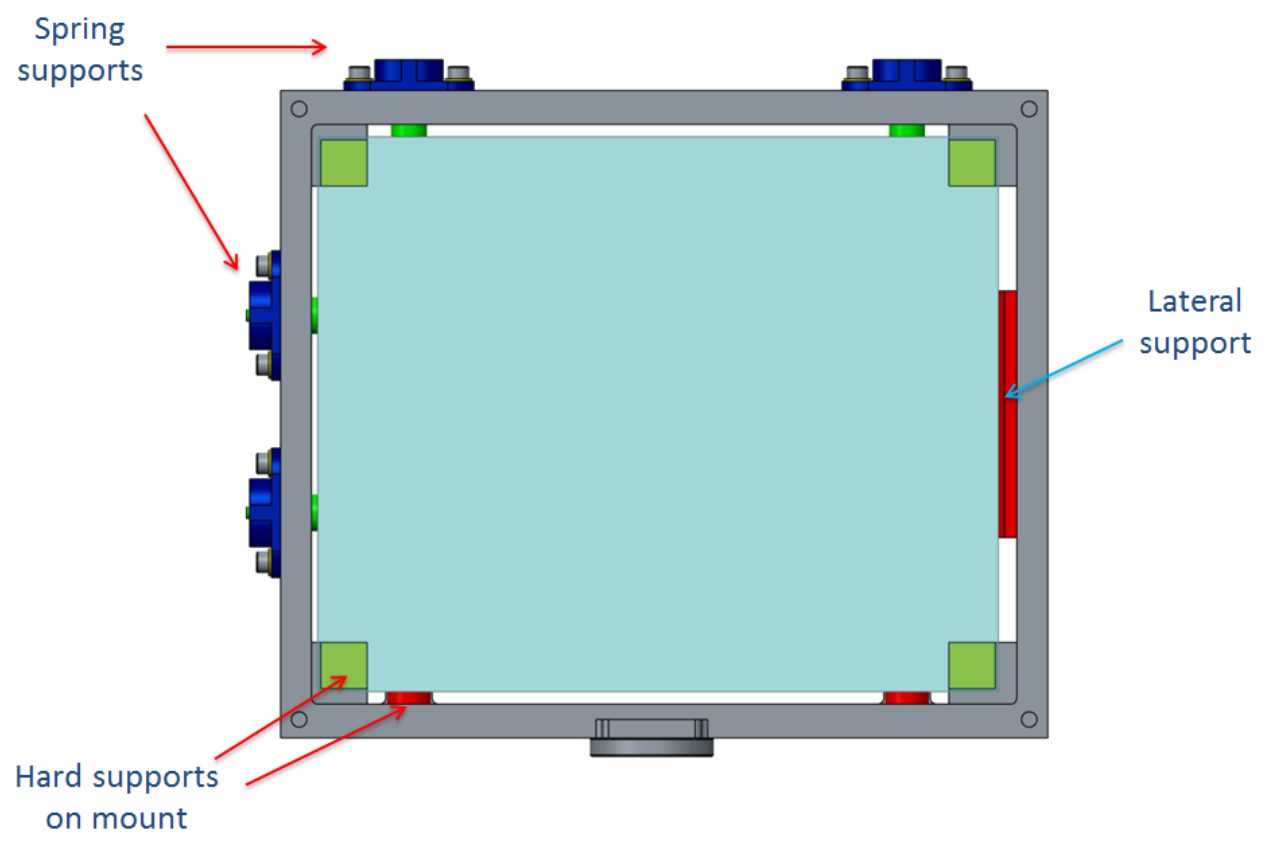

Figure 16: Details of the spring and hard supports of the low resolution VPH mount

\section{$\underline{\text { Status }}$}

At this moment, all LR VPH mounts are assembled, and 4 of them have their gratings inside. There is still one grating at the optical shop.

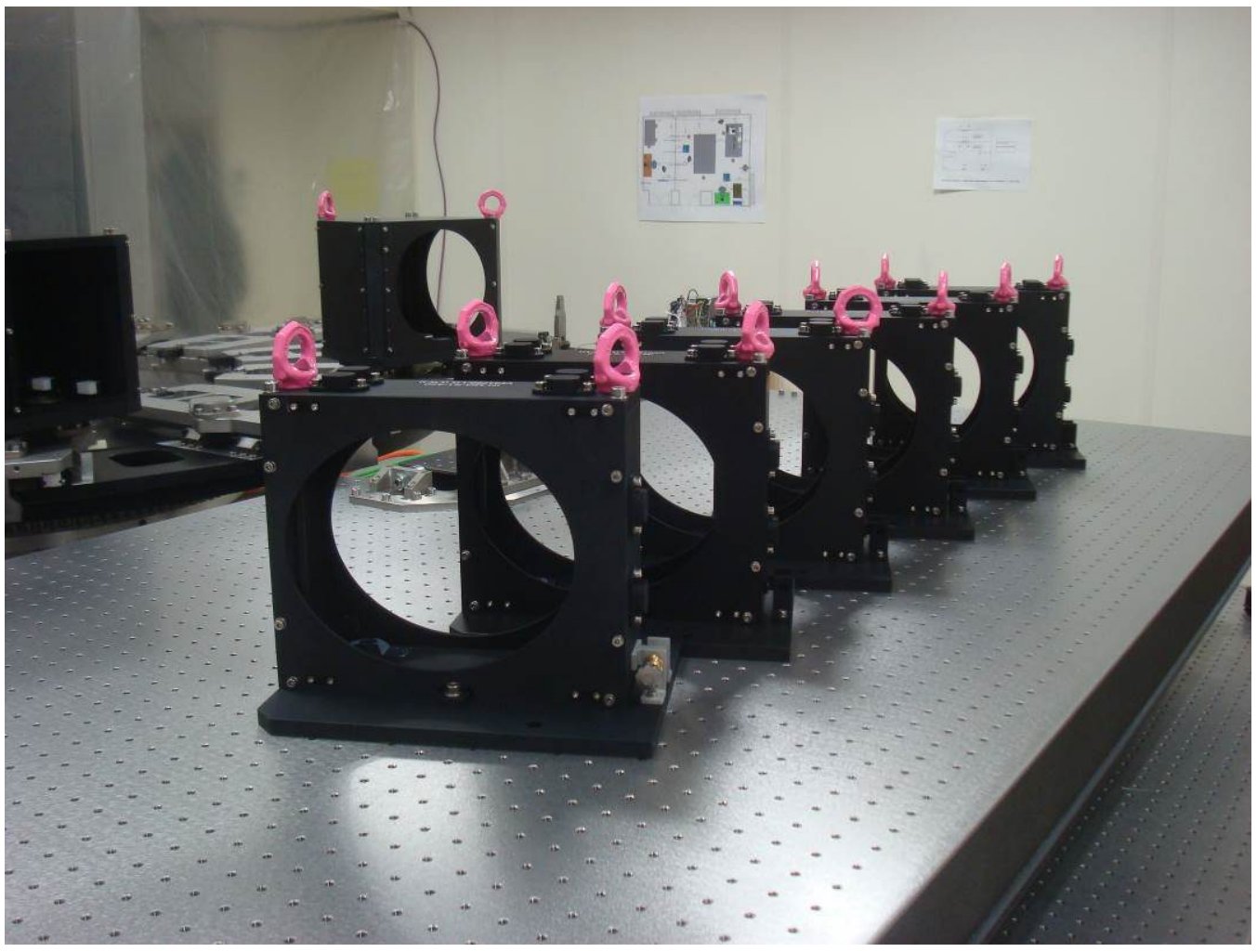

Figure 17: Complete set of LR mounts assembled 


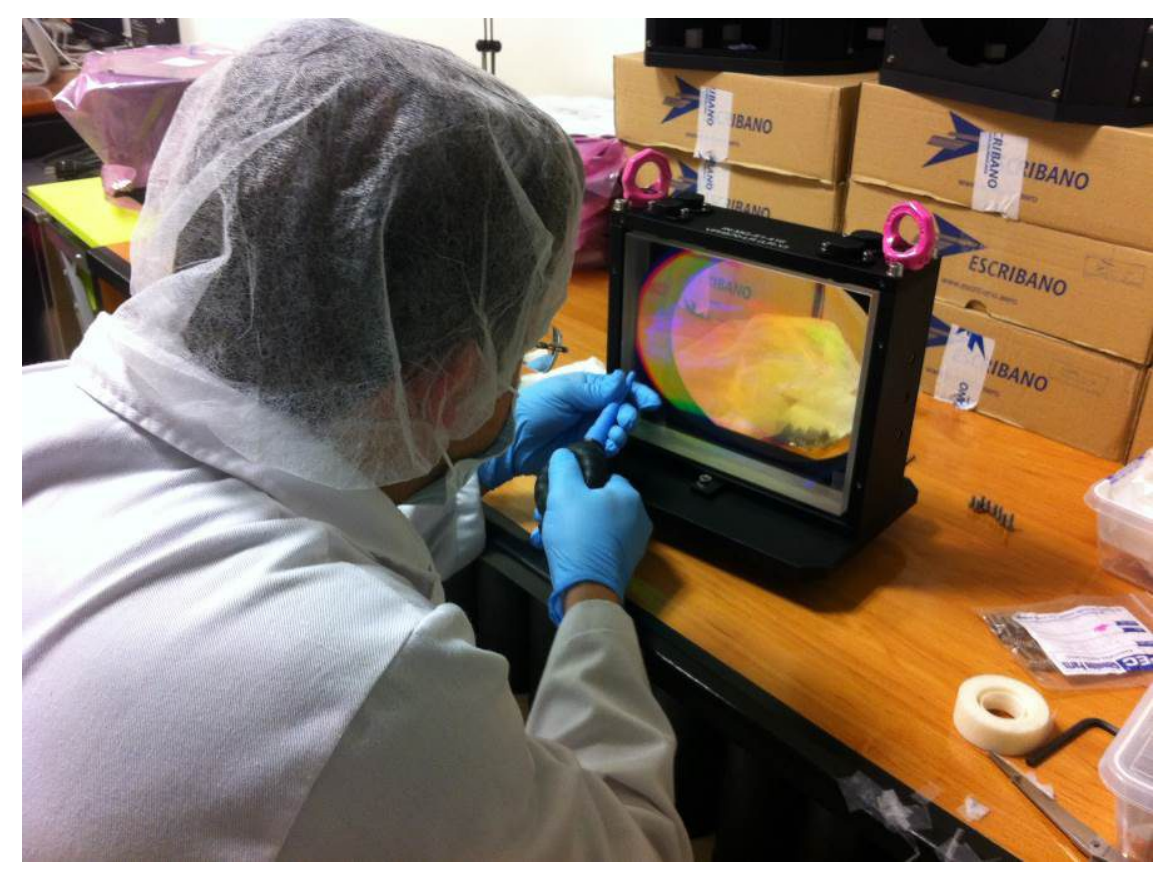

Figure 18: One LR VPH during its integration on its mount

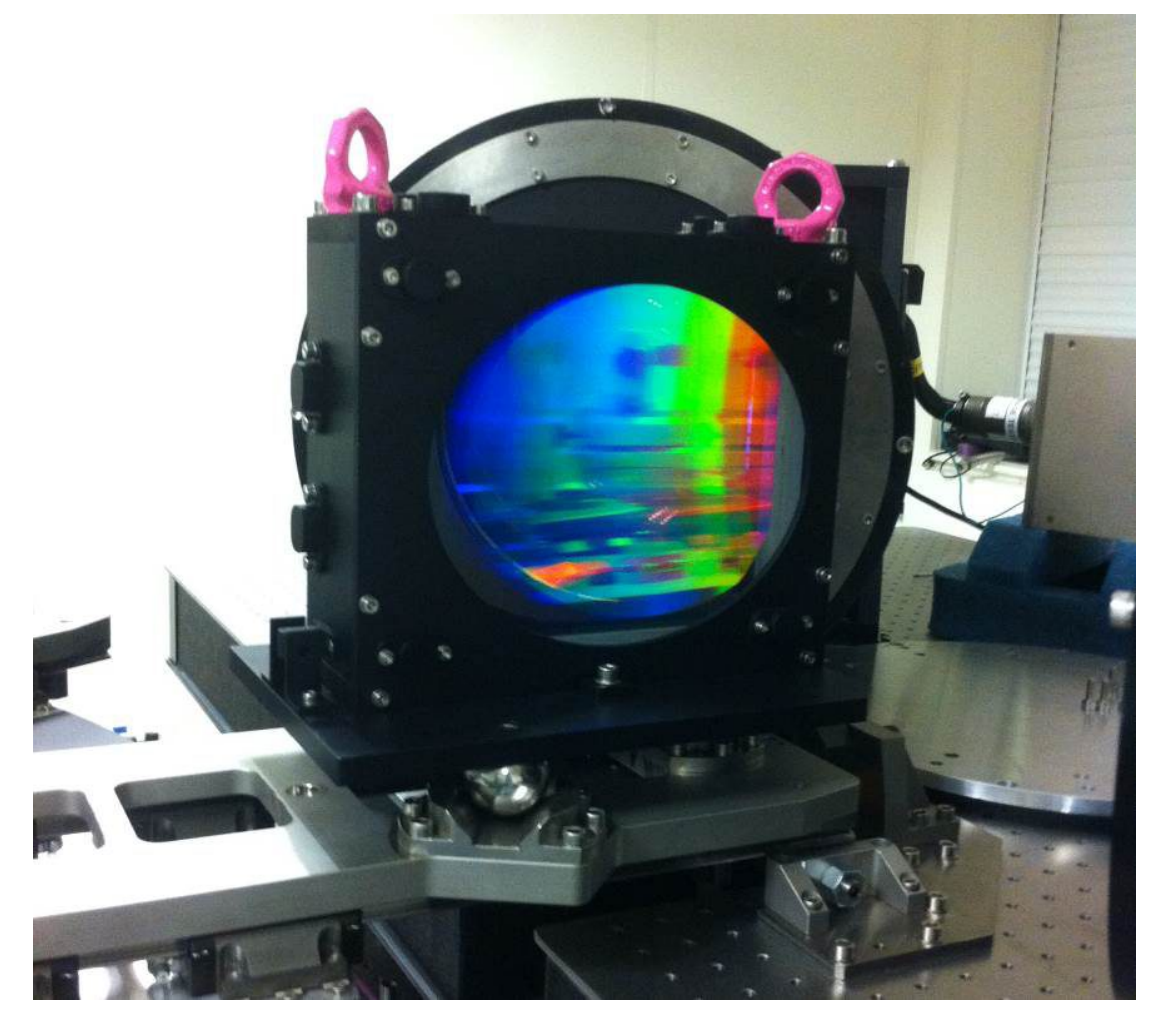

Figure 19: One LR VPH mount on the observing position during AIV. 


\subsubsection{Design of the opto-mechanical mount of the medium and high resolution (MR and HR) VPH}

The medium and high-resolution VPH gratings present a quite different configuration due to the fact that they are provided with the gratings sandwiched between flat windows and two symmetric prisms that allow the beam to incident on the VPH in the optimum angle. These are quite large and massive prisms and are glued to the silica windows with RVT141 silicone. So, we have 4 different optical elements glued to each other.

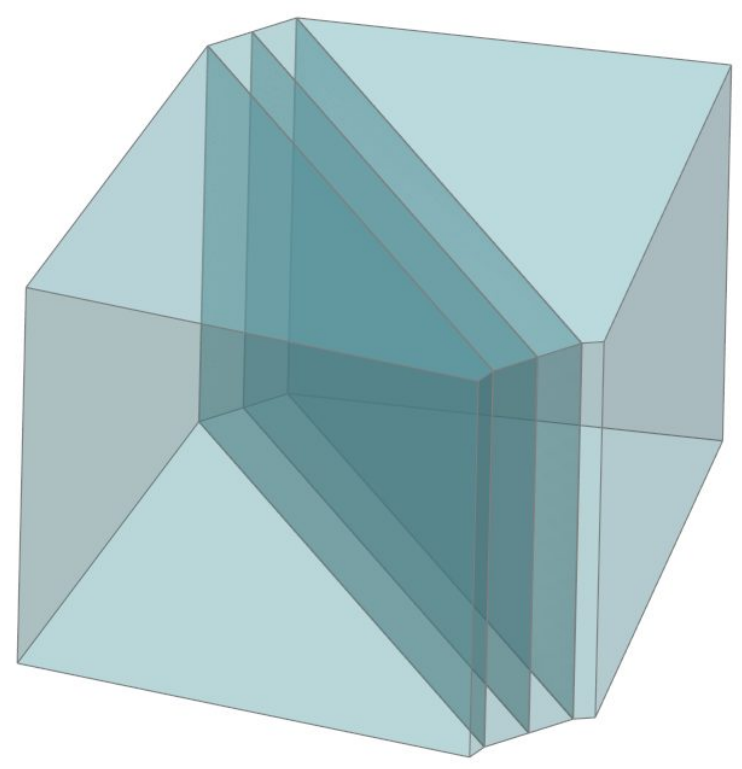

Figure 20: The medium resolution VPH is comprised of two PBM2Y prisms and the gelatin sandwiched between two fused silica $20 \mathrm{~mm}$ thick windows.

The concept is based on a stiff baseplate where the VPH is laid to rest, and a set of pillars that maintain the assembly preloaded with a set of PTFE pads, which has CTE larger than the aluminum of the pillars and the glass of the VPH.The "box" is completed with 4 lateral plates that are screwed to the pillars, 2 of them having the appropriate aperture for the light (baffles) and a top plate.

The baseplate is made of a single part of aluminum alloy (6082-T6) with overall dimensions $356 \times 280 \times 16 \mathrm{~mm}$. As it is shown on Figure 21, it is heavily mechanized to allow the precise location of:

Three circular holes to insert the gimbal supports for the VPHs

Two grooves to insert the axial support for the fused silica windows

Four lateral polymer pads ( 2 for each prism) that provide lateral preload for the prisms.

A fixed reference at the windows that enclose the grating

Four pillars

- $\quad$ Three semi-spheres for the location on the platform. 


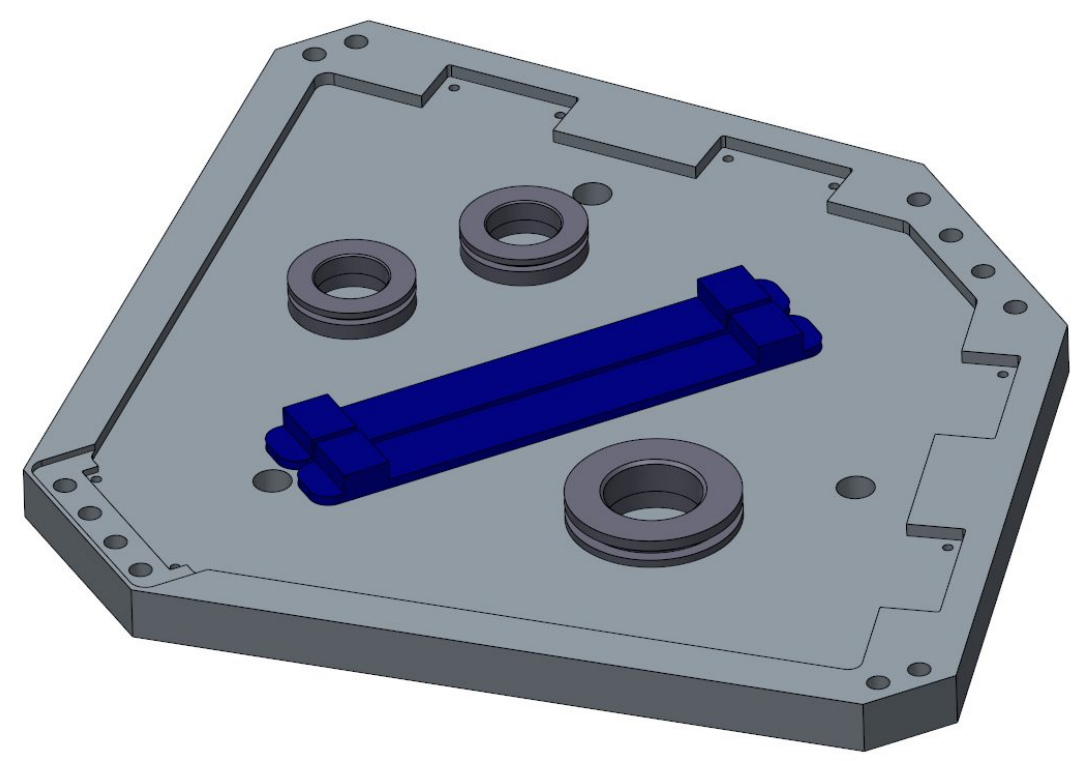

Figure 21: The aluminum baseplate with the supports for the prisms (large spherical washers) and the windows

The main axial support for the MR VPH prisms is comprised of three commercial spherical washers inserted on conical seats. This configuration is selected to ensure that the glued prisms rest on both sides and on the full contact area, even though there is a slight deviation from one side to the other after gluing, could this be on height or slope.

With this "gimbal" configuration, the spherical washer rotates itself to meet the planar surface of the prism. Once the first prism is on a stable position, the whole 4-prism grating is able to rotate around those pair of supports to reach the orientation of the second prism, which will rest on the other (single, larger) spherical washer.

The supports are located around the center of gravity of either prism. In addition to these spherical supports, there is a spring-loaded support under each fused silica window. The part is made of aluminum alloy, the same as the main body. The load of each pair of springs equals the weight of the silica window ( $2 \mathrm{Kg} \mathrm{each}$ ). This is to reduce the shear load on the gelatin and the glued joints.

The location and thickness of the lateral PTFE pads has been calculated taking into account the respective angles of the faces of the prisms and equilibrating the opposite forces. Their mission is to compensate the differential thermal expansions of the aluminum cell $(\mathrm{CTE}=23)$ and the prisms $(\mathrm{CTE}=8.5)$ and maintain the contact and preload. There is also a fixed reference on the silica windows $(\mathrm{CTE}=0.5)$ and the other 4 pads maintain the prisms in contact with the reference. 


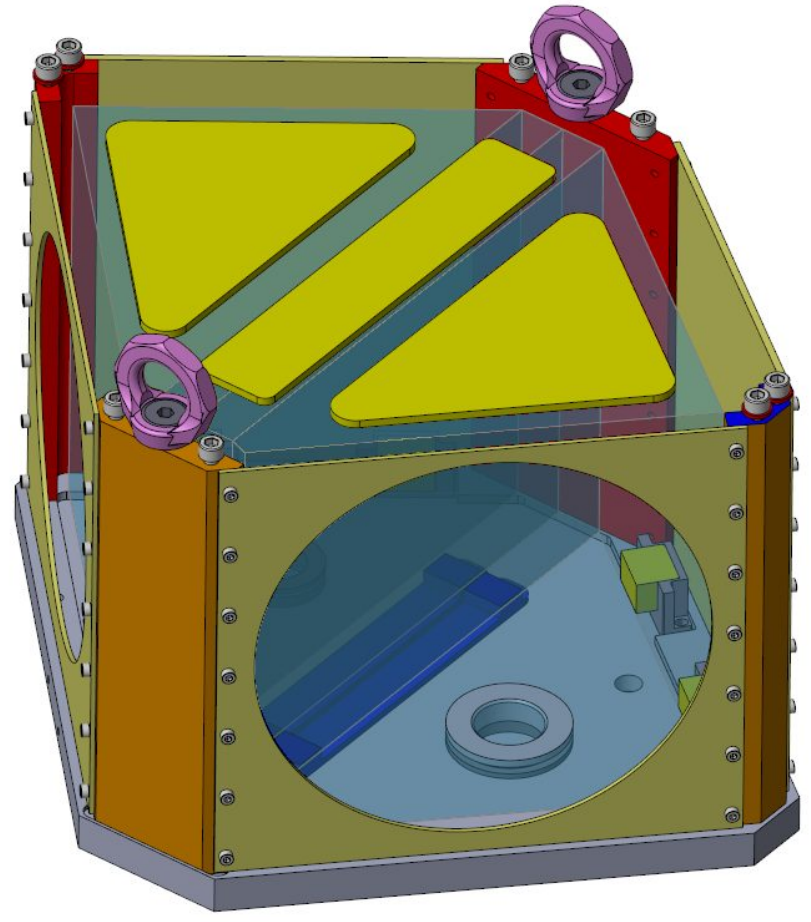

Figure 22: The complete CAD of the MR mount (the top plate is removed)

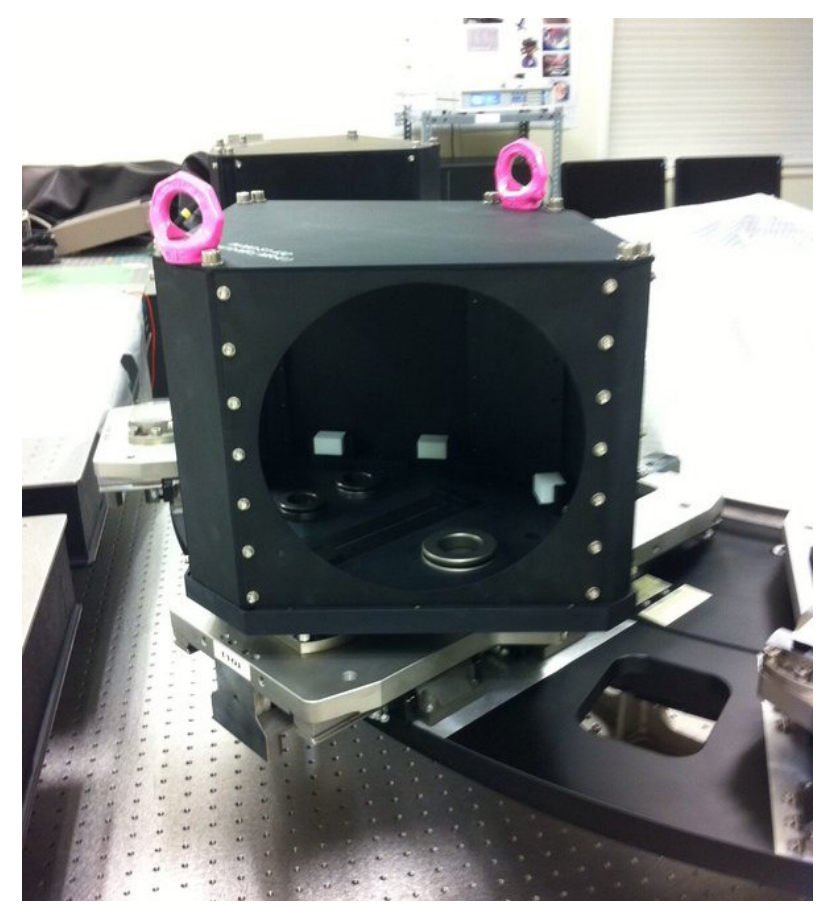

Figure 23: A completely assembled MR mechanical mount presented on the wheel 


\section{Status}

At this moment, all HR mounts have been assembled and two (out of 10) MR mounts are ready to be assembled, with all the parts mechanized. All the prisms are still at the optical shop and will be installed shortly on the mounts.

\subsection{Targets and collimator installation}

Following the alignment procedure, the next step was the installation of two reflecting targets at the position of the observing VPH. The first target was a reflective target placed in the geometrical intersection of the camera and collimator axis. The target was used to define the collimator and camera axis. The second target was used to define the camera-collimator $34^{\circ}$ angle with respect to the grating. At $34^{\circ}$ from the position of the VPH it was defined the collimator axis, placing an alignment telescope to guide the collimator installation.

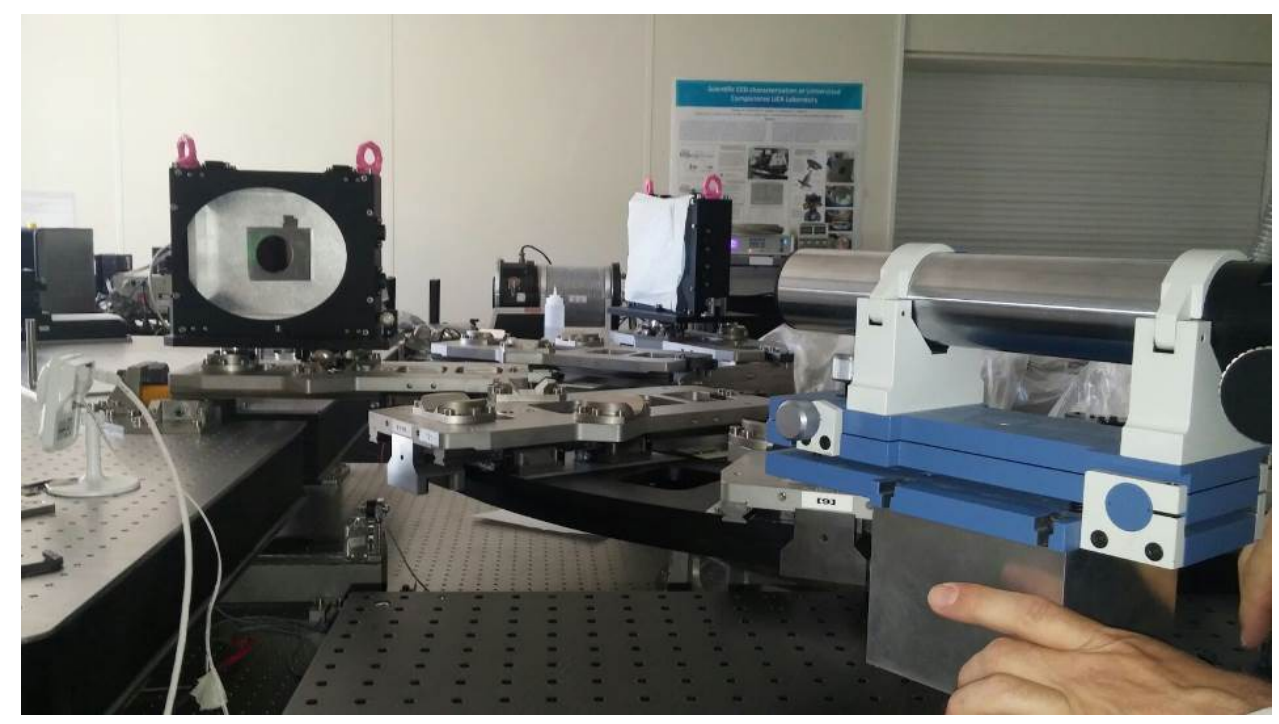

Figure 24: Reflective target and alignment telescope during the alignment

For the installation of the collimator, its stainless steel interface plate was first screwed to the table at the CAD theoretical position. Three rods were screwed to previously defined holes in such a way that the collimator if kept tangent to the rods, it would be placed at its nominal position. And it was. Only a small amount of rotation around the y axis (the vertical) was necessary. 


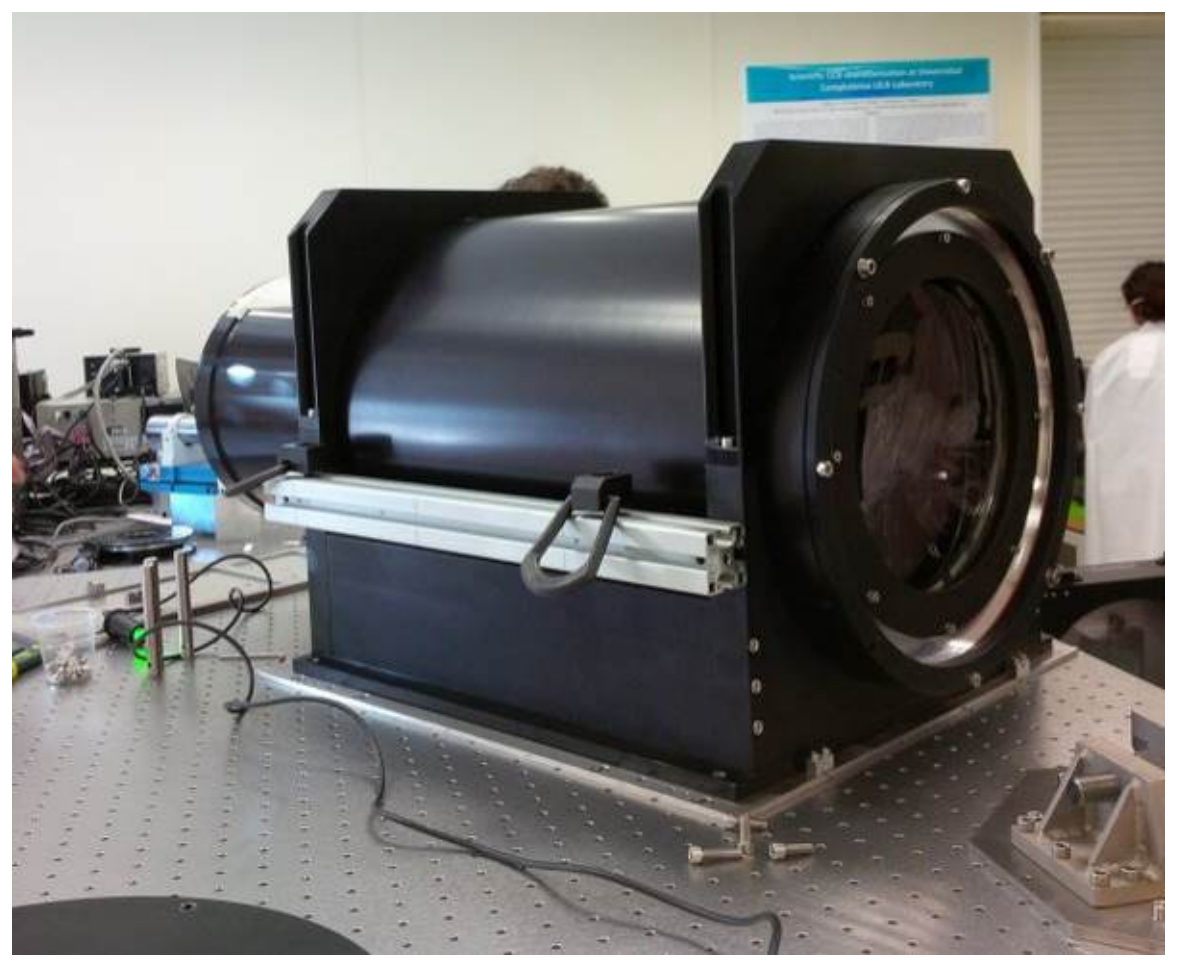

Figure 25: Collimator barrel installed on its position. Notice the stainless interface plate below and one of the rods.

After the collimator, the pseudoslit assembly was installed.

\subsection{Pseudo slit arrangement}

The IFU and the positioners that constitute the Fiber MOS shall be installed at a GTC Folded Cassegrain focal station. Then the fibers bundles shall be routed through the telescope structure and rotators to the Spectrograph entrance. At the entrance there will be a support structure that maintains a calculated distance (to ensure that the bending radius of the fibers is not exceed) from the last stage of the fiber routing to the pseudo-slit assembly. The fibers are aligned in a pseudo-slit. At this position, two orthogonal translation stages are included; one for fiber bundle selection and the second one to allow fine focusing for improving the image quality in the different grating configurations. Due to the fact that the pseudo-slit frames are fixed from below, there must be an intermediate plate that joins the PS unit with the focusing stage. 


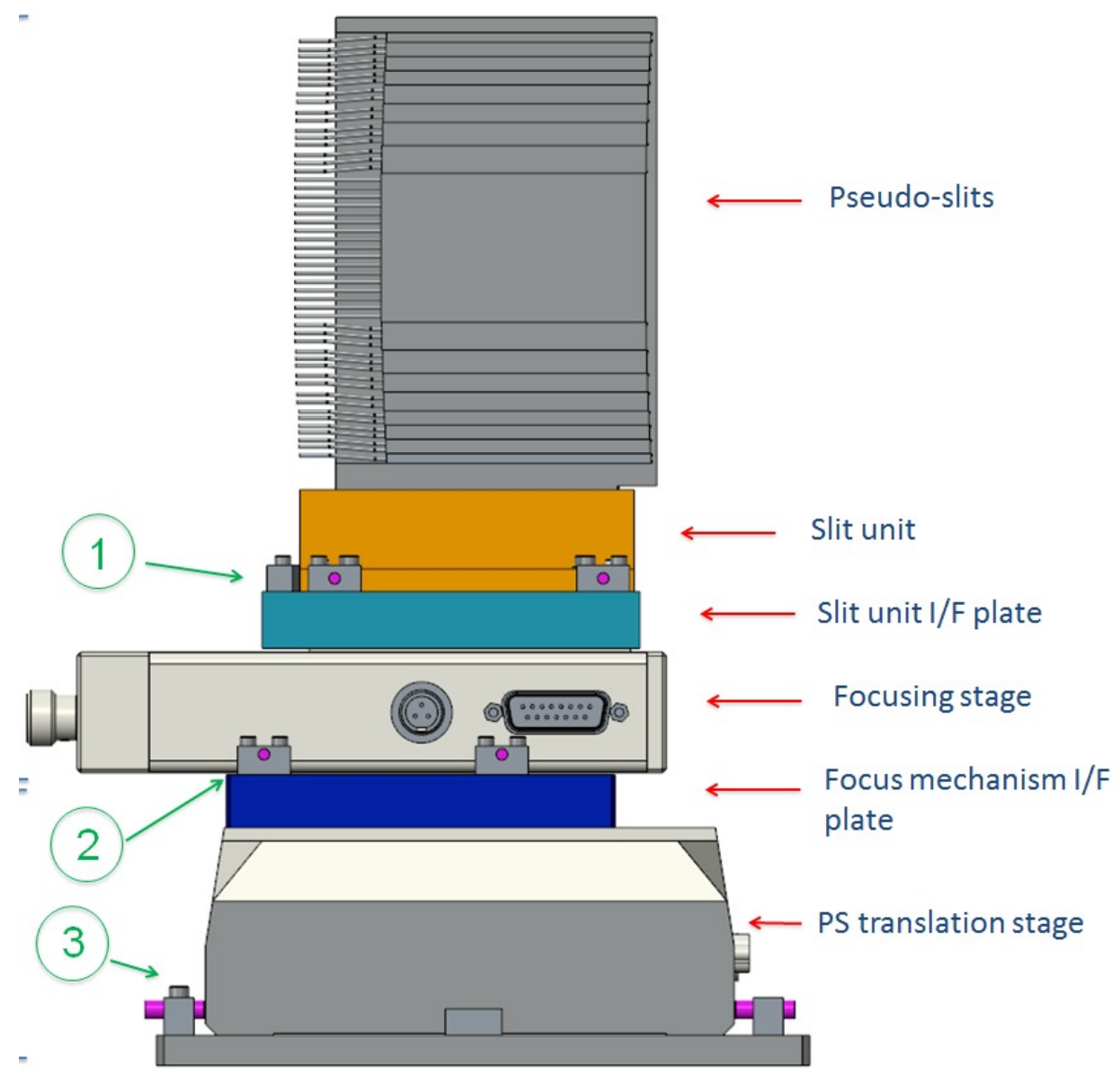

Figure 26: Pseudo-slit assembly elements

\subsubsection{Status}

The pseudoslit assembly was successfully installed on the optical table after the collimator. Both mechanisms were aligned and the precision and repeatability of the movements was confirmed, that is, $1 \mu \mathrm{m}$ for slit interchange and $10 \mu \mathrm{m}$ for focus. The slit change mechanism is M511.2DD from Physik Instrumente, and the focus mechanism is M605.1DD, also from PI. 

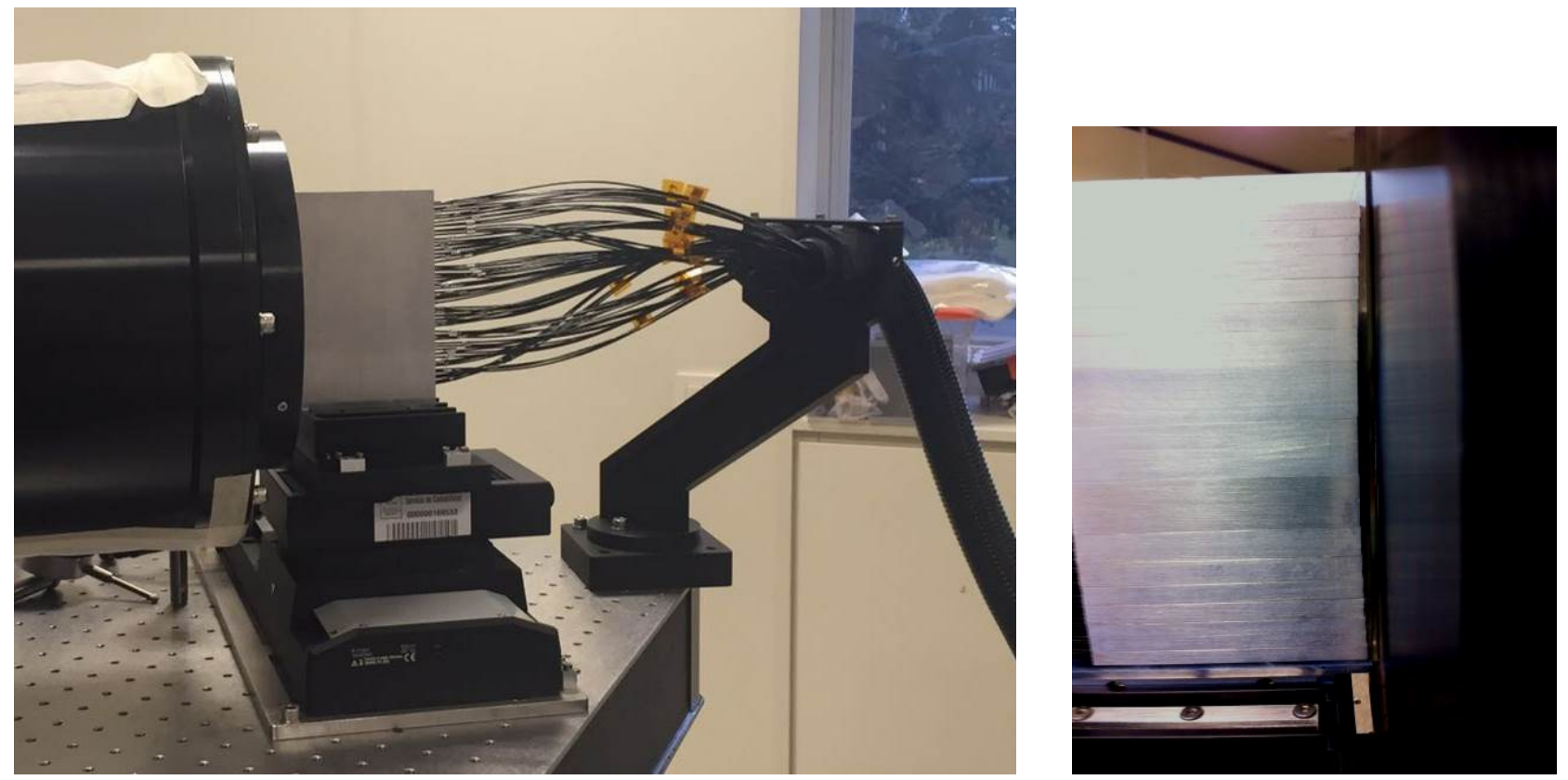

Figure 27: Pseudo-slit arrangement during AIV. Picture on the right shows how close is the pseudoslit to the collimator

As it is in the case of the collimator, there is a 316 stainless steel interface plate under the pseudoslit assembly. This plate is fixed to the table with 8 screws to offer a flat surface (flatness better than $0.2 \mathrm{~mm}$ overall) to attach the pseudoslit large mechanism. When travelling to the observatory, we will place 3 references at this plate to re-install the pseudo-slit assembly.

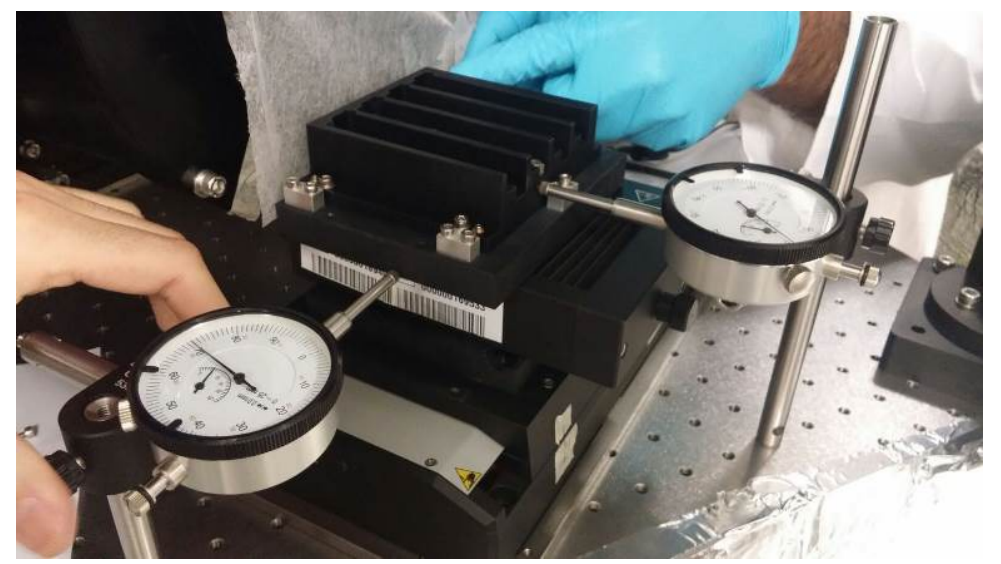

Figure 28: Pseudo-slit arrangement during AIV. Checking parallelism of both stages.

\subsection{Shutter installation}

The shutter is inserted in the collimator barrel, placed after the first lens of the collimator. It is a rotary shutter with a mini-motor that rotates a hexagonal hollow structure. This hexagon is mechanized to allow the light to be blocked, to pass or locate an order sorting filter at $60^{\circ}$ intervals.

Additional components for this mechanism are a pair of angular contact ball bearings to support the load, a motor coupling, locking nuts and a supporting structure for the stepper motor. 


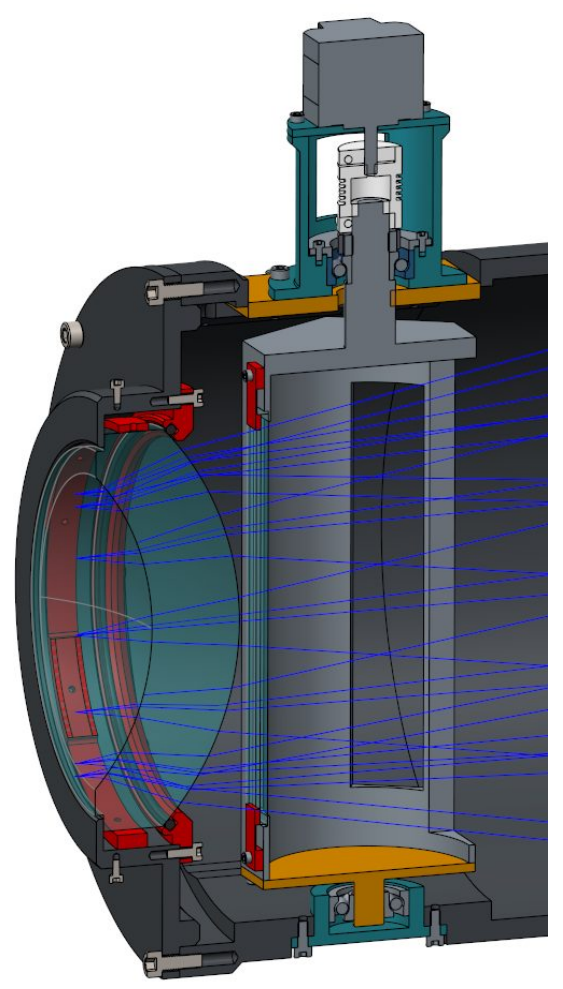

Figure 29: Sectioned view of the shutter located in the collimator barrel.

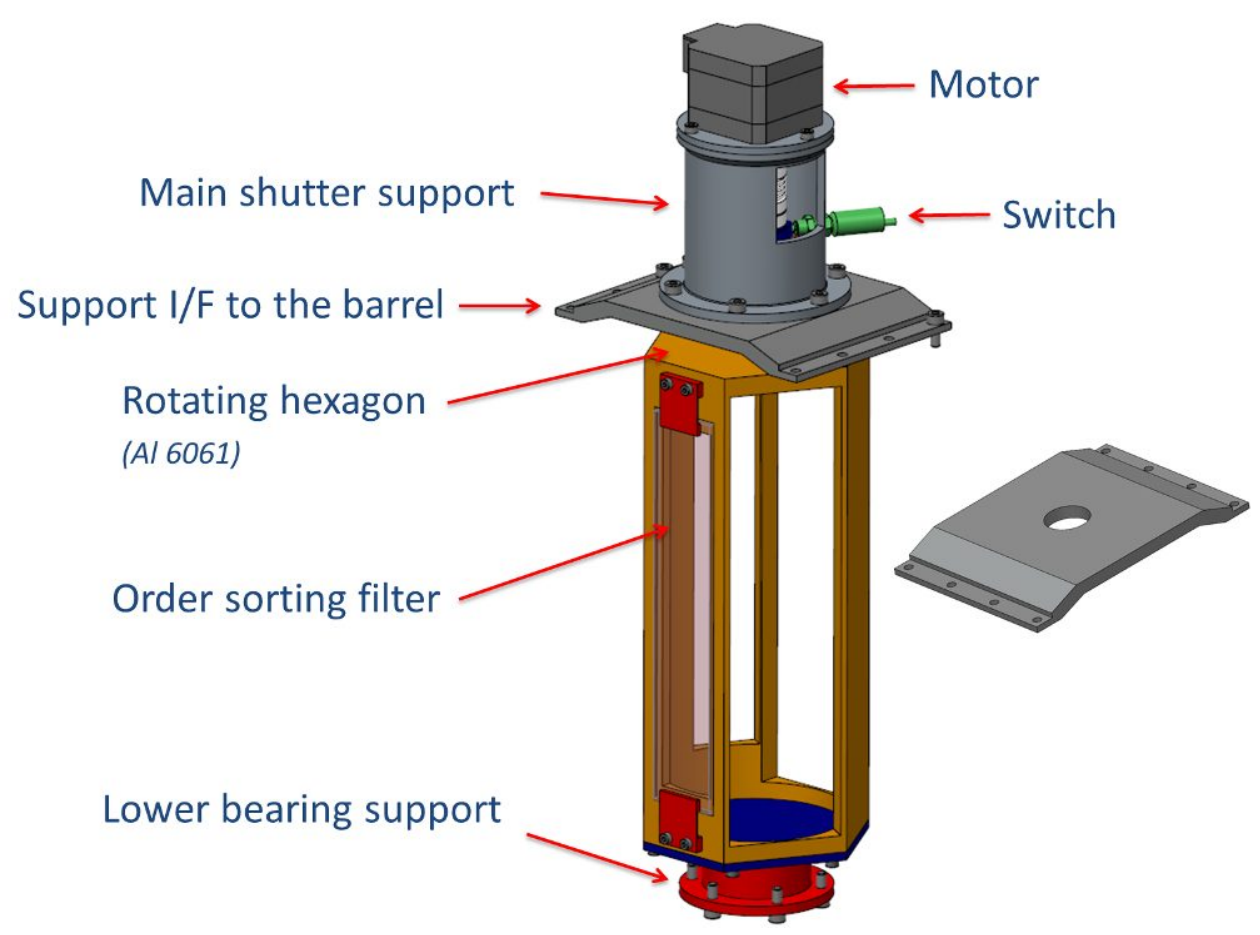

Figure 30: Main elements of the shutter mechanism 


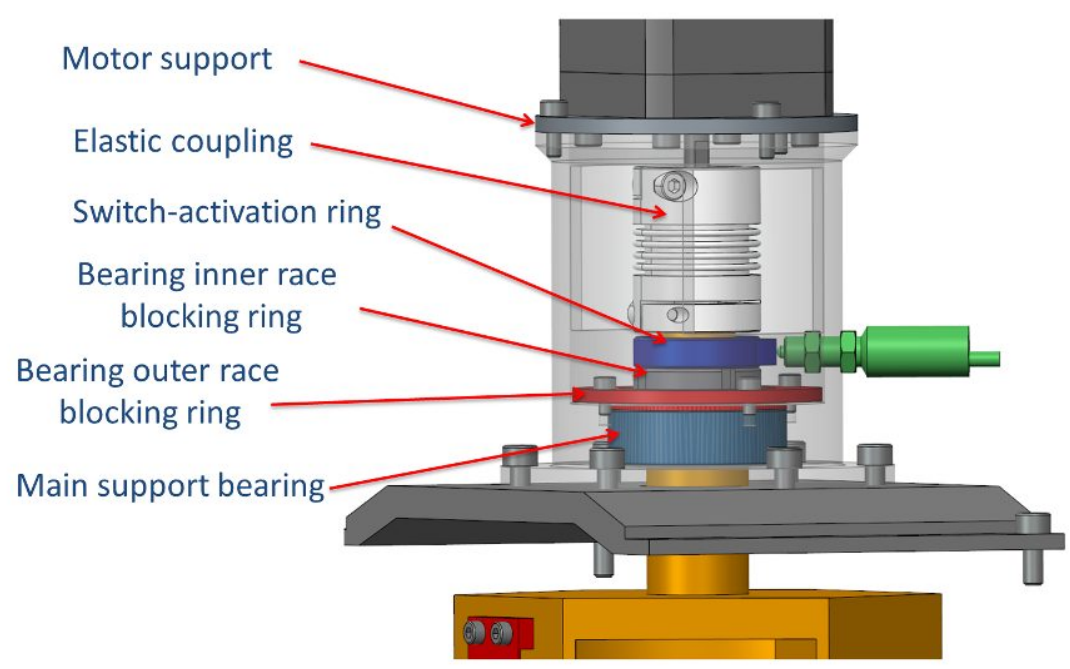

Figure 31: Details of the shutter mechanism

\section{$\underline{\text { Status }}$}

At this moment, the shutter is tested and installed on the collimator.

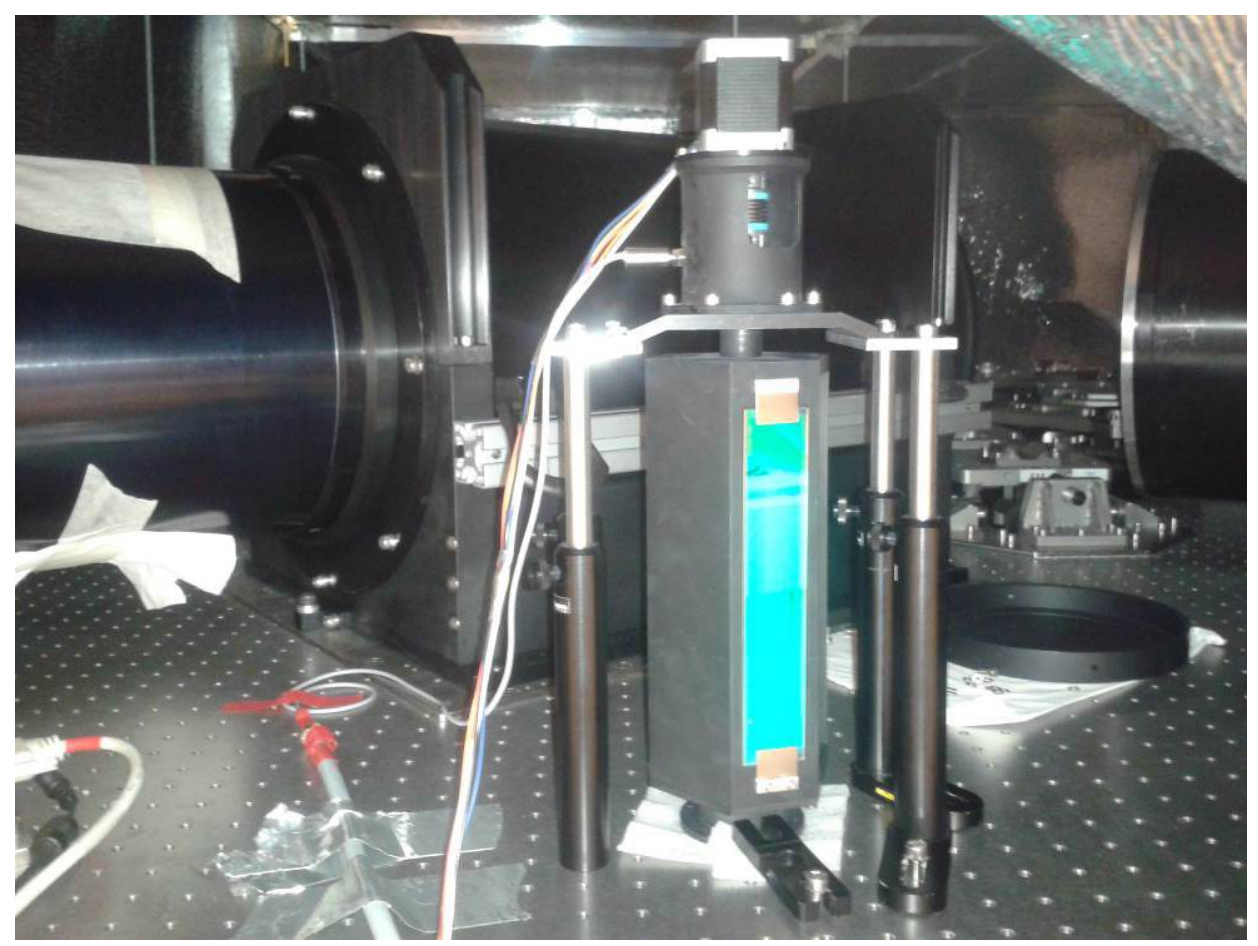

Figure 32: Shutter mechanism being tested before being inserted on its location on the collimator barrel (on its left tape) 


\subsection{Camera-cryostat installation}

In MEGARA spectrograph, the cryostat is attached to the camera. This is common practice that minimizes differential rotations between them. The camera is attached to the aluminum alloy interface structure and this is resting on the table with the intermediate stainless steel plate, as it is the case for the collimator. The camera and cryostat were previously attached together in a clean room and then taken to the LICA lab to be installed on the table. As it was the case for the collimator, three rods guided the assembly support structure to its nominal position. When the CCD was cooled, images were acquired and the next phase of the AIV started.

Figure 33 shows the camera-cryostat installed (on the left) with the collimator and a VPH on the observing position.

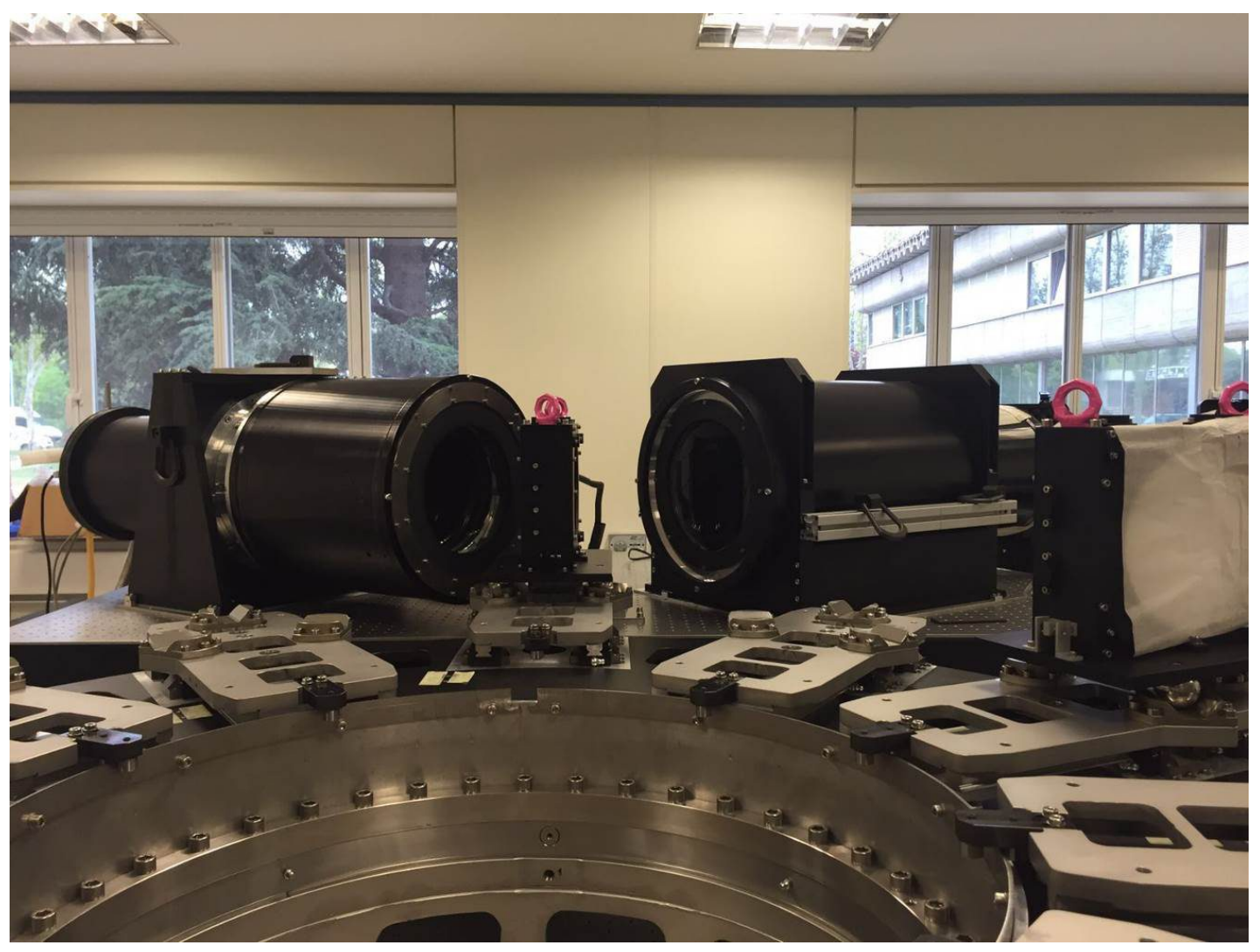

Figure 33: Camera-cryostat and collimator installed on the table during AIV

\subsection{Conclusions}

MEGARA spectrograph was successfully assembled, integrated and is now during its verification campaign. All mechanisms are tested and perform as required, normally better. The laboratory acceptance is scheduled for September 2016. The delivery to the observatory will take place on November 2016 with the installation being currently scheduled for December 2016.

\section{REFERENCES}

[1] Gil de Paz, A., et al., "MEGARA, the new intermediate-resolution optical IFU\&MOS for GTC: getting ready for the telescope", Proc. SPIE 9908-57, (2016). 